\title{
Initial production and fruit quality of fifty-seven sweet orange varieties on four rootstocks in Southern state of Bahia
}

\author{
Stanley Bravo Buffon ${ }^{1}$, Moises Zucoloto ${ }^{2}$, Orlando Sampaio Passos ${ }^{3}$, \\ Dimmy Herllen Silveira Gomes Barbosa ${ }^{4}$, Marcelo Soares Altoé ${ }^{5}$, Andreia Lopes de Morais ${ }^{6}$ \\ Abstract -Prior knowledge of scion/rootstock combinations is essential for establishing appropriate \\ citrus orchard management strategies. The objective of this work was to assess the initial yield and \\ fruit quality among 228 scion/rootstock combinations set up in the municipality of Ibirapuã, extreme \\ south of the state of Bahia in 2015. Harvests were carried out from April to August 2019, when \\ trees reached 4 years of age. Number, total mass and cross-sectional diameter of fruits, productive \\ efficiency, pulp yield, soluble solids content, titratable acidity and SS/AT ratio were evaluated. \\ Statistical difference for all characteristics related to production and fruit quality regarding \\ factors studied and their combinations was observed. Among 'Pera' orange clones, 'Pera CNPMF \\ A-15'/'San Diego' citrandarin and 'Pera CNPMF D-3'/'Riverside' citrandarin combinations stood \\ out. 'Sincorá' orange, in combination with 'San Diego' and 'Indio' citrandarins presented potential \\ to be recommended to producers who wish to anticipate their harvests, as well as 'Natal Ipeal' \\ orange in combination with 'Indio' citrandarin, in the sense of postponing them. \\ Index terms: Citrus sinensis; citrandarin; Citrus sunki; Poncirus trifoliata.

\section{Produção inicial e qualidade de frutos de cinquenta e sete copas de laranjeira-doce sobre quatro porta-enxertos no extremo Sul da Bahia}

Corresponding author: stanleybbuffon@gmail.com

Received: January 26, 2021 Accepted: July 27, 2021

Copyright: All the contents of this journal, except where otherwise noted, is licensed under a Creative Commons Attribution License.

\section{(cc) $\mathrm{EY}$}

Resumo- O conhecimento prévio de combinações copa/porta-enxerto é fundamental para o estabelecimento de estratégias adequadas de manejo de pomares de citros. O objetivo deste trabalho foi avaliar a produtividade e a qualidade iniciais de frutos entre 228 combinações copa/portaenxerto implantadas no Município de Ibirapuã, Extremo Sul da Bahia, em 2015. As colheitas foram realizadas no período de abril a agosto de 2019, quando as plantas atingiram 4 anos. Avaliaram-se o número, a massa total e o diâmetro transversal dos frutos, a eficiência produtiva, o rendimento de polpa, o teor de sólidos solúveis, a acidez titulável e ratio. Houve diferença estatística para todas as características relacionadas à produção e à qualidade dos frutos quanto aos fatores estudados e suas combinações. Dentre os clones de laranjeira 'Pera' estudados, as combinações 'Pera CNPMF A-15'/citrandarin 'San Diego' e 'Pera CNPMF D-3'/citrandarin 'Riverside' foram as que mais se destacaram. A laranjeira 'Sincorá', em combinação com os citrandarins 'San Diego' e 'Indio', tem potencial de recomendação aos produtores que desejam antecipar suas colheitas, assim como a laranjeira 'Natal Ipeal', em combinação com o citrandarin 'Indio', no sentido de postergá-las.

Termos para indexação: Citrus sinensis; citrandarin; Citrus sunki; Poncirus trifoliata.

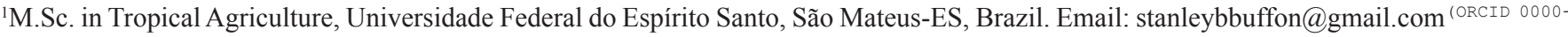
0002-9201-2695)

${ }^{2} \mathrm{PhD}$, Researcher at Universidade Federal do Espírito Santo, Alegre-ES, Brazil. Email: moises.zucoloto@ufes.br (0RCID 0000-0003-0539-4750) ${ }^{3}$ M.Sc., Researcher at Embrapa Mandioca e Fruticultura, Cruz das almas-BA, Brazil. Email: orlando.passos@embrapa.br ${ }^{(0 R C I D} 0000-0002-0851-$ 6541

${ }^{4} \mathrm{PhD}$, Researcher at Embrapa Mandioca e Fruticultura, Cruz das almas-BA, Brazil. Email: dimmy.barbosa@embrapa.br (ORCID 0000-0002-41324226)

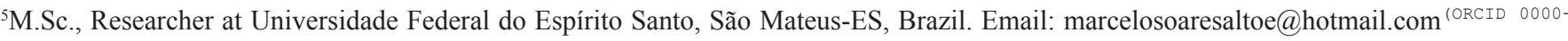
$0002-1842-8289)$

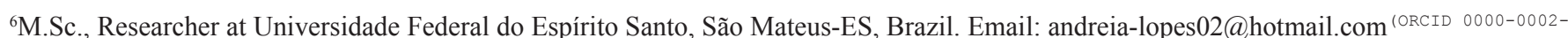
2767-5804)
} 
Citrus are among the most cultivated fruit species in the world, mainly in subtropical and tropical climates. The main citrus product in the Brazilian scenario is intended for industry, for the production of frozen concentrated orange juice for export, of which Brazil accounts for $50 \%$ of the total world production, followed by the United States (FAO, 2015). The Brazilian production of sweet oranges [Citrus sinensis (L.) Osbeck], in 2019, was approximately 17.6 million tons in an approximate area of 608.2 thousand hectares of harvested area, especially in the states of São Paulo. Paulo, Minas Gerais, Paraná and Bahia, with the highest productions, respectively (IBGE, 2020).

Despite the wide variety of genetic material from sweet oranges, the national citrus industry predominantly uses 'Pera' and 'Valencia' orange cultivars on 'Rangpur' and 'Citrumelo Swingle' rootstocks. This makes crops susceptible to losses due to the onset of diseases such as Phytophthora gummosis, citrus tristeza virus and citrus sudden-death (FADEL et al., 2018; RODRIGUES et al., 2016; SOARES; et al., 2015).

With the objective of proposing new rootstock options for the national citrus industry, crosses between 'Sunki' mandarins [Citrus sunki (Hayata) hort. ex Tanaka] and 'Cleopatra (Citrus reshni hort. ex Tanaka) with trifoliata [Poncirus trifoliata (L.) Raf.] have been performed, giving rise to hybrids called citrandarins, which have great potential for use as rootstock. This type of crossing aims to add characteristics present in mandarins, such as less susceptibility to citrus exocortis viroid, citrus decline, citrus tristeza virus and citrus sudden-death. For $P$. trifoliata, studies seek to add resistance to Phytophthora gummosis and citrus tristeza virus, adaptation to low temperatures and soils subject to flooding, in addition to inducing size reduction to scion varieties grafted on them, which characteristic is verified particularly in 'Flying Dragon' cultivar (POMPEU JUNIOR; BLUMER, 2009). Among mandarins, 'Tropical Sunki' stands out, which has as main characteristics high number of seeds per fruit, polyembryony degree close to $100 \%$ and greater tolerance to Phytophthora gummosis, compared to other selections of this mandarin (SOARES FILHO et al., 2002).

To achieve success in the citrus activity, with fruit yield and quality, it is not enough just to analyze the different scions of sweet oranges and rootstocks alone, but their combinations. These interactions can induce behavioral variations, making it necessary to study scion/rootstock combinations. Another factor that suffers interference and is directly linked to the success of the scion/rootstock combination refers to fruit production, which may also vary according to the interaction of the genotype with the environment (SCHÄFER et al., 2001; PRUDENTE; SILVA, 2006). Therefore, prior knowledge of rootstock combinations with orange scions is important to establish crop management strategies. In this sense, the objective of this study was to identify among 228 scion/rootstock combinations those that present the best productive aspects and fruit quality in the extreme south of the state of Bahia.

The experiment was carried out in an experimental area of Fazenda Chão Bello, municipality of Ibirapuã,

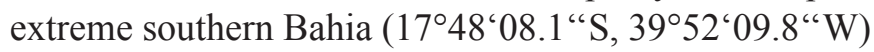
and altitude of $95 \mathrm{~m}$ a.s.l. The climate is classified as tropical humid or sub-humid (Am), according to the Köppen classification, with average annual temperature of $23.6{ }^{\circ} \mathrm{C}$ (ALVARES et al., 2013). Average monthly rainfall was $76.3 \mathrm{~mm}$ between January 2018 and December 2019, according to data collected from the rain gauge installed in the experimental area.

The soil chemical characterization in the $0-20$ $\mathrm{cm}$ layer, according to the soil analysis methodology of Embrapa (2009), was: $\mathrm{pH}$ in water $=5.2(1: 2.5$ ratio); phosphorus $=12 \mathrm{mg} / \mathrm{dm}^{3}$; potassium $=51 \mathrm{mg} /$ $\mathrm{dm}^{3} ;$ sodium $=15 \mathrm{mg} / \mathrm{dm}^{3} ;$ calcium $=1.5 \mathrm{cmol} / \mathrm{dm}^{3}$; magnesium $=0.3 \mathrm{cmol} / \mathrm{dm}^{3} ;$ aluminum $=0.4 \mathrm{cmol} / \mathrm{dm}^{3}$; $\mathrm{H}+\mathrm{Al}=3.4 \mathrm{cmolc} / \mathrm{dm}^{3}$; sum of bases $=2.0 \mathrm{cmolc} / \mathrm{dm}^{3}$; effective cation exchange capacity $=2.4 \mathrm{cmolc} / \mathrm{dm}^{3}$; base saturation index $=37 \%$; sodium saturation index $=$ $1.1 \%$; organic matter $=1.88 \mathrm{dag} / \mathrm{dm}^{3}$. For phosphorus, potassium and sodium, the Mehlich-1 extractor was used; for calcium, magnesium and aluminum, $\mathrm{KCl}$ at concentration of $1 \mathrm{~mol} / \mathrm{L}$ and for $\mathrm{H}+\mathrm{Al}$, SMP extractor.

A completely randomized design was used. The scheme adopted was a $4 \times 57$ factorial, consisting of four rootstocks combined with 57 sweet orange varieties, with 3 replicates and 2 plants per plot. Seedlings were produced in nursery at "Embrapa Mandioca e Fruticultura" in Cruz das Almas-BA. The orange cultivars evaluated were: 'Pera' (CNPMF 01, 02, A-15, B-12, C-21, C-32, D-3, D-6, D-9, D-12, D-25, E-3 and E-6, Olímpia, Bianchi, GE-03, Vacinada, and Ibotirama selections), 'Natal' (CNPMF 01, 02 and 112, Ipeal and Folha Murcha selections), 'Valencia' (CNPMF 01, 02, 03 , 21, 27, 36, F-11, Midknight, Criola, Delta, Late, L. Shaffey, Chapman, L. White, Montemorelos, Registro, Tuxpan selections), 'Bern', 'Jaffa', 'F-Menuda', 'Sincorá', 'Aquiri', 'Early Oblong', 'Russas PS', 'Selecta de Itaboraí', 'Salustiana', 'Pineapple', 'Westin', 'Diva', 'Hamlin CNPMF-20', 'Crescent sweet', 'Melrosa' and 'Flor de Brumadinho'. With the exception of 'Pera' GE03, Bianchi, Olímpia and Vacinada selections that came from the "Sylvio Moreira" Citriculture Center (IAC), the others came from "Embrapa Mandioca e Fruticultura", with 'Pera', 'Natal' and 'Valencia' selections being nuclear clones obtained and evaluated by the Eastern Agricultural Research Institute (IPEAL), agency preceding Embrapa. Scion varieties were grafted onto 'Sunki Tropical' (TST) mandarins and 'Indio' (IND), 'Riverside' (RIV) and 'San Diego' (SD) citrandarins ( $C$. 
sunki $\times P$. trifoliata), selected by the Citrus Breeding Program at "Embrapa Mandioca e Fruticultura".

The experiment was installed in the field on $04 / 12 / 2015$ with spacing of $6 \mathrm{~m}$ between rows and $3 \mathrm{~m}$ between plants. The irrigation system used was microsprinkler with flow of $72 \mathrm{~L} / \mathrm{h}$, in two fixed irrigation shifts of $6 \mathrm{~mm} /$ day. Fertilization was carried out according to recommendations from the partner citrus grower. For phytosanitary control, fungicides and insecticides registered for the crop were used, according to periodic monitoring in the area. To control weeds in planting rows, herbicides based on glyphosate and glufosinate were applied. Cultural treatments such as mowing between rows and plant pruning were carried out in accordance with the producer's management and to standards recommended for the crop.

In 2018, due to the low yield, harvest was carried out only with the purpose of verifying the harvest time of the different scion/rootstock combinations. Based on 2018 data, harvests were carried out from April to August 2019, evaluating the following morphoagronomic parameters: total number of fruits (NTF, per plant), total fruit weight (MTF, kg/plant), plant height (ALT, m), mean scion diameter in the row direction (DL, $m$ ) and between rows (DE, m). Fruits were manually collected, counted, placed in collection boxes suitable for transport and weighed on scale installed in the experimental area. ALT and scion diameters (DL and DE) were measured using measuring tape graduated in centimeters, and data were used to calculate the scion volume $\left(\mathrm{VC}, \mathrm{m}^{3}\right)$, obtained according to Zekri et al. (2003), by the formula:

\section{$\mathrm{VC}=(\pi / 6) \times \mathrm{ALT} \times \mathrm{DL} \times \mathrm{DE}$}

where: $\mathrm{ALT}=$ plant height $(\mathrm{m}) ; \mathrm{DL}=$ scion diameter in the row direction; $\mathrm{DE}=$ scion diameter $(\mathrm{m})$ perpendicular to the planting row.

Productive efficiency per plant (EP, kg. $\left.\mathrm{m}^{-3}\right)$ was obtained by the relationship between MTF and VC.

For physicochemical evaluations, samples of six fruits per experimental plot were used, taken from the middle third of scions, packed in polyethylene bags, identified and taken to the laboratory. The following characteristics were evaluated: cross-sectional diameter (DT, mm) measured with digital caliper; juice yield (REND, \%) through industrial blender, measuring the percentage of the difference between sample and waste masses (bagasse and seeds); soluble solids content (SS, ${ }^{\circ}$ Brix) with digital refractometer; titratable acidity (AT, \% citric acid) determined by volumetry and using phenolphthalein solution as indicator; and the SS/AT ratio.
Data were submitted to analysis of variance (ANOVA) and the Scott-Knott test was applied to compare treatments at $5 \%$ probability using the $\mathrm{R}$ software (R DEVELOPMENT CORE TEAM, 2011).

All characteristics related to fruit production were significant in terms of the interaction between scion and rootstock (Table 1). Thus, it was observed, as reported by Chaparro-Zambrano et al. (2015), that the rootstock has direct influence on the production of sweet orange scions.

Table 1. Summary of the analysis of variance with source of variation (FV), degree of freedom (GL), mean square $(\mathrm{QM})$ and coefficient of variation $(\mathrm{CV})$ for total number of fruits (NTF), total fruit mass (MTF) and productive efficiency (EP) of 57 sweet orange varieties [Citrus sinensis (L.) Osbeck] in combination with four rootstocks.

\begin{tabular}{ccccc}
\hline \multirow{2}{*}{ FV } & GL & \multicolumn{4}{c}{ QM } \\
\cline { 3 - 5 } PE & 3 & $1303045^{* *}$ & $75724^{* *}$ & $127.747^{* *}$ \\
E & 56 & $757831^{* *}$ & $26488^{* *}$ & $39.912^{* *}$ \\
PE $\times$ E & 168 & $7404435^{* *}$ & $2094 * *$ & $3.892 * *$ \\
RES & 447 & 20141 & 1008 & 0.849 \\
\hline CV $(\%)$ & & 37.74 & 33.98 & 31.59 \\
\hline
\end{tabular}

** Significant at $1 \%$ and $5 \%$ probability by the $\mathrm{F}$ test; $\mathrm{PE}=$ Rootstock; $\mathrm{E}$ $=$ Graft RES $=$ Residue

Scion varieties that presented the highest number of fruits per plant on the 'Sunki Tropical' (TST) mandarin were: 'Pera' CNPMF 01, B-12, D-6, D-9, E-6 and 'Natal' CNPMF 01 (Table 2). On 'San Diego' (SD) citrandarin were 'Pera' CNPMF E-6, E-3, 'Olimpia' and 'Sincorá'. For 'Pera' CNPMF 01, D-3, E-3, 'Olimpia', 'Bianchi', 'GE-03', 'Natal' CNPMF 01 and 02, higher number of fruits per plant was observed on 'Riverside' (RIV) citrandarin and for 'Pera' GE-03 and 'Sincorá' on 'Indio' (IND) citrandarin. Among combinations that most produced fruits, TST and RIV rootstocks stand out, which together accounted for more than $60 \%$ of averages. Cultivars with higher NTF in two of the rootstocks were 'Pera' CNPMF 01 and E-6, 'Olimpia', 'GE-03', 'Sincorá' and 'Natal' CNPMF 01. 
Table 2. Total number of fruits per plant of 228 scion/rootstock combinations comprising 57 sweet orange varieties [Citrus sinensis (L.) Osbeck] on 'Sunki Tropical' (TST) mandarin rootstocks [Citrus sunki (Hayata) hort. ex Tanaka] and 'San Diego' (SD), 'Riverside' (RIV) and 'Indio' (IND) citrandarins [C. sunki × Poncirus trifoliata (L.) Raf.].

\begin{tabular}{|c|c|c|c|c|}
\hline \multirow{2}{*}{ Scion variety } & \multicolumn{4}{|c|}{ Rootstock } \\
\hline & TST & SD & RIV & IND \\
\hline Aquiri & $76 \mathrm{Ac}$ & $142 \mathrm{Ac}$ & $131 \mathrm{Ac}$ & $171 \mathrm{Ad}$ \\
\hline Berna & $4 \mathrm{Bc}$ & $62 \mathrm{Bc}$ & $141 \mathrm{Ac}$ & $149 \mathrm{Ad}$ \\
\hline Crescent sweet & $76 \mathrm{Bc}$ & $115 \mathrm{Bc}$ & $203 \mathrm{Ac}$ & $84 \mathrm{Be}$ \\
\hline Diva & $11 \mathrm{Bc}$ & $22 \mathrm{Bc}$ & $201 \mathrm{Ac}$ & $27 \mathrm{Be}$ \\
\hline Early Oblong & $62 \mathrm{Bc}$ & $67 \mathrm{Bc}$ & $367 \mathrm{Ab}$ & $172 \mathrm{Bd}$ \\
\hline F-Menuda & $1 \mathrm{Ac}$ & $6 \mathrm{Ac}$ & $25 \mathrm{Ad}$ & $30 \mathrm{Ae}$ \\
\hline Flor de Brumadinho & $59 \mathrm{Ac}$ & $96 \mathrm{Ac}$ & $140 \mathrm{Ac}$ & $155 \mathrm{Ad}$ \\
\hline Hamlin CNPMF 20 & $227 \mathrm{Bb}$ & $185 \mathrm{Bc}$ & $375 \mathrm{Ab}$ & $312 \mathrm{Ac}$ \\
\hline Jaffa & $16 \mathrm{Bc}$ & $28 \mathrm{Bc}$ & $194 \mathrm{Ac}$ & $134 \mathrm{Ad}$ \\
\hline Mel Rosa & $56 \mathrm{Bc}$ & $232 \mathrm{Ab}$ & $135 \mathrm{Bc}$ & $139 \mathrm{Bd}$ \\
\hline Natal CNPMF 01 & $387 \mathrm{Aa}$ & $253 \mathrm{Bb}$ & $442 \mathrm{Aa}$ & $315 \mathrm{Bc}$ \\
\hline Natal CNPMF 02 & $154 \mathrm{Cc}$ & $319 \mathrm{Bb}$ & $468 \mathrm{Aa}$ & $343 \mathrm{Bc}$ \\
\hline Natal CNPMF 112 & $203 \mathrm{Bb}$ & $135 \mathrm{Bc}$ & $338 \mathrm{Ab}$ & $297 \mathrm{Ac}$ \\
\hline Natal Folha Murcha & $3 \mathrm{Ac}$ & $42 \mathrm{Ac}$ & $3 \mathrm{Ad}$ & $18 \mathrm{Ae}$ \\
\hline Natal Ipeal & $240 \mathrm{Bb}$ & $299 \mathrm{Bb}$ & $397 \mathrm{Ab}$ & $270 \mathrm{Bc}$ \\
\hline Pera Bianchi & $300 \mathrm{Bb}$ & $299 \mathrm{Bb}$ & $497 \mathrm{Aa}$ & $397 \mathrm{Ab}$ \\
\hline Pera CNPMF 01 & $384 \mathrm{Aa}$ & $281 \mathrm{Bb}$ & $466 \mathrm{Aa}$ & $294 \mathrm{Bc}$ \\
\hline Pera CNPMF 02 & $234 \mathrm{Ab}$ & $328 \mathrm{Ab}$ & $301 \mathrm{Ab}$ & $348 \mathrm{Ac}$ \\
\hline Pera CNPMF A-15 & $242 \mathrm{Ab}$ & $213 \mathrm{Ab}$ & $210 \mathrm{Ac}$ & $205 \mathrm{Ad}$ \\
\hline Pera CNPMF B-12 & $482 \mathrm{Aa}$ & $231 \mathrm{Bb}$ & $403 \mathrm{Ab}$ & $347 \mathrm{Ac}$ \\
\hline Pera CNPMF C-21 & $266 \mathrm{Bb}$ & $255 \mathrm{Bb}$ & $409 \mathrm{Ab}$ & $257 \mathrm{Bd}$ \\
\hline Pera CNPMF C-32 & $146 \mathrm{Bc}$ & $139 \mathrm{Bc}$ & $339 \mathrm{Ab}$ & $193 \mathrm{Bd}$ \\
\hline Pera CNPMF D-3 & $324 \mathrm{Bb}$ & $269 \mathrm{Bb}$ & $488 \mathrm{Aa}$ & $287 \mathrm{Bc}$ \\
\hline Pera CNPMF D-6 & $386 \mathrm{Aa}$ & $227 \mathrm{Bb}$ & $417 \mathrm{Ab}$ & $168 \mathrm{Bd}$ \\
\hline Pera CNPMF D-9 & $407 \mathrm{Aa}$ & $280 \mathrm{Ab}$ & $387 \mathrm{Ab}$ & $320 \mathrm{Ac}$ \\
\hline Pera CNPMF D-12 & $331 \mathrm{Ab}$ & $175 \mathrm{Bc}$ & $350 \mathrm{Ab}$ & $329 \mathrm{Ac}$ \\
\hline Pera CNPMF D-25 & $319 \mathrm{Ab}$ & $309 \mathrm{Ab}$ & $382 \mathrm{Ab}$ & $352 \mathrm{Ac}$ \\
\hline Pera CNPMF E-3 & $317 \mathrm{Bb}$ & $394 \mathrm{Ba}$ & $565 \mathrm{Aa}$ & $466 \mathrm{Ab}$ \\
\hline Pera CNPMF E-6 & $472 \mathrm{Aa}$ & $430 \mathrm{Aa}$ & $343 \mathrm{Ab}$ & $424 \mathrm{Ab}$ \\
\hline Pera GE-03 & $346 \mathrm{Bb}$ & $334 \mathrm{Bb}$ & $455 \mathrm{Aa}$ & $561 \mathrm{Aa}$ \\
\hline Pera de Ibotirama & $4 \mathrm{Ac}$ & $64 \mathrm{Ac}$ & $109 \mathrm{Ad}$ & $59 \mathrm{Ae}$ \\
\hline Pera Olímpia & $341 \mathrm{Ab}$ & $445 \mathrm{Aa}$ & $505 \mathrm{Aa}$ & $411 \mathrm{Ab}$ \\
\hline Pera Vacinada & $316 \mathrm{Bb}$ & $292 \mathrm{Bb}$ & $365 \mathrm{Bb}$ & $445 \mathrm{Ab}$ \\
\hline Pineapple & $18 \mathrm{Cc}$ & $153 \mathrm{Bc}$ & $309 \mathrm{Ab}$ & $202 \mathrm{Bd}$ \\
\hline Russas P.S. & $21 \mathrm{Bc}$ & $98 \mathrm{Bc}$ & $245 \mathrm{Ac}$ & $98 \mathrm{Be}$ \\
\hline Salustiana & $3 \mathrm{Ac}$ & $71 \mathrm{Ac}$ & $136 \mathrm{Ac}$ & $16 \mathrm{Ae}$ \\
\hline Seleta de Itaboraí & $24 \mathrm{Ac}$ & $145 \mathrm{Ac}$ & $168 \mathrm{Ac}$ & $145 \mathrm{Ad}$ \\
\hline Sincorá & $277 \mathrm{Bb}$ & $456 \mathrm{Aa}$ & $393 \mathrm{Ab}$ & $519 \mathrm{Aa}$ \\
\hline Valencia Chapman & $232 \mathrm{Ab}$ & $242 \mathrm{Ab}$ & $205 \mathrm{Ac}$ & $165 \mathrm{Ad}$ \\
\hline Valencia CNPMF & $35 \mathrm{Ac}$ & $137 \mathrm{Ac}$ & $145 \mathrm{Ac}$ & $69 \mathrm{Ae}$ \\
\hline Valencia CNPMF 01 & $16 \mathrm{Ac}$ & $38 \mathrm{Ac}$ & $45 \mathrm{Ad}$ & $47 \mathrm{Ae}$ \\
\hline Valencia CNPMF 02 & $34 \mathrm{Ac}$ & $123 \mathrm{Ac}$ & $188 \mathrm{Ac}$ & $83 \mathrm{Ae}$ \\
\hline Valencia CNPMF 03 & $14 \mathrm{Ac}$ & $99 \mathrm{Ac}$ & $108 \mathrm{Ad}$ & $56 \mathrm{Ae}$ \\
\hline Valencia CNPMF 21 & $80 \mathrm{Bc}$ & $98 \mathrm{Bc}$ & $132 \mathrm{Bc}$ & $299 \mathrm{Ac}$ \\
\hline Valencia CNPMF 27 & $35 \mathrm{Ac}$ & $136 \mathrm{Ac}$ & $157 \mathrm{Ac}$ & $110 \mathrm{Ae}$ \\
\hline Valencia CNPMF 36 & $48 \mathrm{Ac}$ & $119 \mathrm{Ac}$ & $188 \mathrm{Ac}$ & $91 \mathrm{Ae}$ \\
\hline Valencia CNPMF F-11 & $69 \mathrm{Bc}$ & $127 \mathrm{Bc}$ & $213 \mathrm{Ac}$ & $74 \mathrm{Be}$ \\
\hline Valencia Criola & $17 \mathrm{Ac}$ & $152 \mathrm{Ac}$ & $145 \mathrm{Ac}$ & $86 \mathrm{Ae}$ \\
\hline Valencia Delta & $46 \mathrm{Ac}$ & $134 \mathrm{Ac}$ & $113 \mathrm{Ad}$ & $65 \mathrm{Ae}$ \\
\hline Valencia L. Shaffey & $22 \mathrm{Ac}$ & $62 \mathrm{Ac}$ & $171 \mathrm{Ac}$ & $67 \mathrm{Ae}$ \\
\hline Valencia L. White & $120 \mathrm{Ac}$ & $142 \mathrm{Ac}$ & $173 \mathrm{Ac}$ & $52 \mathrm{Ae}$ \\
\hline Valencia Late & $173 \mathrm{Ac}$ & $42 \mathrm{Ac}$ & $114 \mathrm{Ad}$ & $72 \mathrm{Ae}$ \\
\hline Valencia Midknight & $15 \mathrm{Ac}$ & $38 \mathrm{Ac}$ & $48 \mathrm{Ad}$ & $26 \mathrm{Ae}$ \\
\hline Valencia Montemorelos & $91 \mathrm{Ac}$ & $169 \mathrm{Ac}$ & $247 \mathrm{Ac}$ & $159 \mathrm{Ad}$ \\
\hline Valencia Registro & $119 \mathrm{Bc}$ & $102 \mathrm{Bc}$ & $331 \mathrm{Ab}$ & $201 \mathrm{Bd}$ \\
\hline Valencia Tuxpan & $20 \mathrm{Ac}$ & $29 \mathrm{Ac}$ & $85 \mathrm{Ad}$ & $115 \mathrm{Ae}$ \\
\hline Westin & $8 \mathrm{Bc}$ & $157 \mathrm{Ac}$ & $142 \mathrm{Ac}$ & $111 \mathrm{Ae}$ \\
\hline
\end{tabular}

Means followed by the same uppercase letter in the row and lowercase in the column do not differ by the Scott-Knott mean cluster test at 5\% probability. 
The combinations that presented averages above 500 fruits/plant were related to 'Pera Olímpia' and 'Pera CNPMF E-3' cultivars on 'Riverside' citrandarin and 'Pera GE-03' and 'Sincorá' on 'Indio' citrandarin. Among 'Natal' orange selections, 'Natal CNPMF-01' stood out in number of fruits in combination with 'Sunki Tropical' mandarin (387 fruits) and 'Riverside' citrandarin (442), as well as 'Natal CNPMF- 02' also on this citrandarin (468). Although preliminary, these results suggest that 'Pera' and 'Natal' selections are efficient in fruit production, being superior compared to 'Pera' orange evaluated by Rodrigues et al. (2019) in combination with nine rootstocks in the state of Acre.

In relation to total fruit mass (MTF), the combination between 'San Diego' citrandarin and 'Sincorá' orange showed greater expression of this character (Table 3). It is noteworthy that in this scion, higher MTF values were observed in the four rootstocks (Figure 1), with emphasis on 'San Diego' and 'Indio' citrandarins (115.64 and $111.1 \mathrm{~kg} / \mathrm{plant})$ with average masses per fruit of 254 and $214 \mathrm{~g}$ respectively, superior to results found by Coelho et al. (2019), with average mass for 'Pera Rio' cultivar, in conventional system, of 204 g per fruit. 'Pera Olímpia/ 'Riverside' citrandarin and 'Pera GE-03/ Indio' citrandarin combinations also presented MTF above $100 \mathrm{~kg} / \mathrm{plant}$; however, lower averages, of 200 and $184 \mathrm{~g}$ per fruit, respectively. Based on observed data, 'Sincorá' orange can be an alternative to 'Pera' and 'Natal' oranges in extreme south of the Bahia. However, future evaluations should be carried out to confirm or not these results.

Taking into account the spacing adopted in this study and according to IBGE (2020), $40.35 \%$ of scion/ rootstock combinations evaluated presented yield higher than the national average of 28.96 t.ha $^{-1}$ in 2019 , above $52.08 \mathrm{~kg} /$ plant. 'Riverside' citrandarin stood out, which induced fruit yields above $60 \mathrm{~kg}$ per plant in all 'Pera' orange selections, according to Bastos et al. (2014), justifying its excellent behavior when grafted with sweet orange trees. It is noteworthy that yields presented here were obtained with 4.5-year-old plants.

It should be noted that greater amount of fruits may reflect greater total fruit mass, as observed, since of the 19 combinations that had the highest average NTF, 15 also presented higher MTF than the others. However, these characteristics may not indicate increase in productive gains, since, in their totality, small fruits, non-commercial standard and vigorous plants with excessive growth are taken into account. Thus, NTF and MTF should not be used solely as a way of selecting the most advantageous combinations, requiring evaluating other variables related to fruit production and quality, such as productive efficiency, total acidity and soluble solids content of produced fruits.
The analysis of Table 4 allows verifying that, with regard to productive efficiency (EP), it was found that, on 'Sunki Tropical' mandarin, scion varieties that presented the highest EP were 'Pera CNPMF-01', 'Pera CNPMF B-12', 'Pera CNPMF-D3', 'Pera CNPMF D-6', 'Pera CNPMF D-9', 'Pera CNPMF E-6', Pera Olímpia' and 'Pera GE-03'. Regarding 'Pera CNPMF-02', 'Pera CNPMF A-15' and 'Pera Olímpia' cultivars, the highest average EP values were verified for 'San Diego' citrandarin. 'Riverside' citrandarin provided higher EP values on 'Pera CNPMF D-3' and 'Pera Olímpia' cultivars, while 'Indio' citrandarin was related to higher yield efficiencies when scion varieties were 'Pera CNPMF E-6', 'Pera Olímpia', 'Pera GE-03' and 'Natal Ipeal'.

Among combinations with the highest EP values, 'Pera CNPMF-02' and 'Pera CNPMF A-15' on 'San Diego' citrandarin stand out, with 9.29 and $10.88 \mathrm{~kg}$ of fruits. $\mathrm{m}^{-3}$ of scion, respectively. These 'Pera' orange selections did not show statistically significant differences for total fruit mass among rootstocks studied, the same not being true in relation to productive efficiency. This fact, as observed by Carvalho et al. (2016), relates to the effect of the rootstock on the size or volume that induces to scion variety, verifying that smaller scions, which have smaller amount of fruits per plant compared to larger scions, can, on the other hand, show greater fruit production per scion volume unit, that is, high fruit production efficiency.

'Pera Olímpia' orange stood out for presenting the highest EP value on the four rootstocks evaluated, notably in relation to 'San Diego', 'Riverside' and 'Indio' citrandarins, with $9.23,8.56$ and $9.23 \mathrm{~kg}$ of fruits.m ${ }^{-3}$ scion, respectively. These values are in line with those obtained by Carvalho et al. (2016) in 'Pera CNPMF-D6' orange scions on these citrandarins. There is, therefore, evidence that these rootstocks have great potential for commercial use in the extreme south of the state of Bahia in combination with 'Pera' orange. 
Table 3. Total fruit mass (kg/plant) of 228 scion/rootstock combinations comprising 57 sweet orange varieties [Citrus sinensis (L.) Osbeck] on 'Sunki Tropical' (TST) mandarin rootstocks [Citrus sunki (Hayata) hort. ex Tanaka] and 'San Diego' (SD), 'Riverside' (RIV) and 'Indio' (IND) citrandarins [C. sunki $\times$ Poncirus trifoliata (L.) Raf.].

\begin{tabular}{|c|c|c|c|c|}
\hline \multirow{2}{*}{ Scion variety } & \multicolumn{4}{|c|}{ Rootstock } \\
\hline & TST & $\mathrm{SD}$ & RIV & IND \\
\hline Aquiri & $23.61 \mathrm{Ac}$ & $46.12 \mathrm{Ac}$ & $44.86 \mathrm{Ac}$ & $54.84 \mathrm{Ab}$ \\
\hline Berna & $0.73 \mathrm{Bd}$ & $18.38 \mathrm{Bd}$ & $38.32 \mathrm{Ac}$ & $40.84 \mathrm{Ac}$ \\
\hline Crescent sweet & $9.13 \mathrm{Ad}$ & $26.04 \mathrm{Ad}$ & $41.95 \mathrm{Ac}$ & $17.74 \mathrm{Ad}$ \\
\hline Diva & $3.95 \mathrm{Bd}$ & $8.34 \mathrm{Bd}$ & $65.44 \mathrm{Ab}$ & $10.81 \mathrm{Bd}$ \\
\hline Early Oblong & $15.26 \mathrm{Bd}$ & $17.71 \mathrm{Bd}$ & $87.02 \mathrm{Aa}$ & $39.01 \mathrm{Bc}$ \\
\hline F-Menuda & $0.18 \mathrm{Ad}$ & $1.70 \mathrm{Ad}$ & $7.61 \mathrm{Ae}$ & $8.66 \mathrm{Ad}$ \\
\hline Flor de Brumadinho & $22.75 \mathrm{Ac}$ & $23.53 \mathrm{Ad}$ & $32.66 \mathrm{Ad}$ & $32.01 \mathrm{Ac}$ \\
\hline Hamlin CNPMF 20 & $54.19 \mathrm{Bb}$ & $50.26 \mathrm{Bc}$ & $97.26 \mathrm{Aa}$ & $76.40 \mathrm{Aa}$ \\
\hline Jaffa & $5.18 \mathrm{Bd}$ & $9.18 \mathrm{Bd}$ & $54.42 \mathrm{Ac}$ & $40.16 \mathrm{Ac}$ \\
\hline Mel Rosa & $19.65 \mathrm{Ac}$ & 43.61 Ac & $51.37 \mathrm{Ac}$ & $42.69 \mathrm{Ac}$ \\
\hline Natal CNPMF 01 & $83.82 \mathrm{Aa}$ & $64.48 \mathrm{Ab}$ & $94.10 \mathrm{Aa}$ & $70.98 \mathrm{Ab}$ \\
\hline Natal CNPMF 02 & $35.13 \mathrm{Bc}$ & $74.00 \mathrm{Ab}$ & $78.63 \mathrm{Ab}$ & $99.01 \mathrm{Aa}$ \\
\hline Natal CNPMF 112 & $48.09 \mathrm{Bb}$ & $39.63 \mathrm{Bc}$ & $71.10 \mathrm{Ab}$ & $77.88 \mathrm{Aa}$ \\
\hline Natal Folha Murcha & $1.06 \mathrm{Ad}$ & $10.54 \mathrm{Ad}$ & $1.15 \mathrm{Ae}$ & $5.99 \mathrm{Ad}$ \\
\hline Natal Ipeal & $50.25 \mathrm{Ab}$ & $54.43 \mathrm{Ac}$ & $76.16 \mathrm{Ab}$ & $56.55 \mathrm{Ab}$ \\
\hline Pera Bianchi & $62.65 \mathrm{Bb}$ & $61.09 \mathrm{Bc}$ & $96.23 \mathrm{Aa}$ & $81.70 \mathrm{Aa}$ \\
\hline Pera CNPMF 01 & $77.83 \mathrm{Aa}$ & $63.52 \mathrm{Ab}$ & $93.10 \mathrm{Aa}$ & $60.93 \mathrm{Ab}$ \\
\hline Pera CNPMF 02 & $43.09 \mathrm{Ab}$ & $70.68 \mathrm{Ab}$ & $62.27 \mathrm{Ab}$ & $69.94 \mathrm{Ab}$ \\
\hline Pera CNPMF A-15 & $51.63 \mathrm{Ab}$ & $42.01 \mathrm{Ac}$ & $60.39 \mathrm{Ac}$ & $57.14 \mathrm{Ab}$ \\
\hline Pera CNPMF B-12 & $92.73 \mathrm{Aa}$ & $50.87 \mathrm{Bc}$ & $86.10 \mathrm{Aa}$ & $71.56 \mathrm{Ab}$ \\
\hline Pera CNPMF C-21 & $59.22 \mathrm{Bb}$ & $46.70 \mathrm{Bc}$ & $82.66 \mathrm{Aa}$ & $58.63 \mathrm{Bb}$ \\
\hline Pera CNPMF C-32 & $34.78 \mathrm{Bc}$ & $34.58 \mathrm{Bd}$ & $73.44 \mathrm{Ab}$ & $51.42 \mathrm{Bb}$ \\
\hline Pera CNPMF D-3 & $63.41 \mathrm{Bb}$ & $56.01 \mathrm{Bc}$ & $91.67 \mathrm{Aa}$ & $69.21 \mathrm{Bb}$ \\
\hline Pera CNPMF D-6 & 86.61 Aa & $53.17 \mathrm{Bc}$ & $85.98 \mathrm{Aa}$ & $42.98 \mathrm{Bc}$ \\
\hline Pera CNPMF D-9 & $78.70 \mathrm{Aa}$ & $58.96 \mathrm{Ac}$ & $84.95 \mathrm{Aa}$ & $73.68 \mathrm{Aa}$ \\
\hline Pera CNPMF D-12 & $61.10 \mathrm{Ab}$ & $38.71 \mathrm{Ac}$ & $70.13 \mathrm{Ab}$ & $67.26 \mathrm{Ab}$ \\
\hline Pera CNPMF D-25 & $82.66 \mathrm{Aa}$ & $80.34 \mathrm{Ab}$ & $94.28 \mathrm{Aa}$ & $84.10 \mathrm{Aa}$ \\
\hline Pera CNPMF E-3 & $59.60 \mathrm{Bb}$ & $77.61 \mathrm{Ab}$ & $99.93 \mathrm{Aa}$ & $86.26 \mathrm{Aa}$ \\
\hline Pera CNPMF E-6 & $87.78 \mathrm{Aa}$ & $74.81 \mathrm{Ab}$ & $63.97 \mathrm{Ab}$ & $80.77 \mathrm{Aa}$ \\
\hline Pera GE-03 & $75.01 \mathrm{Ba}$ & $68.16 \mathrm{Bb}$ & $95.22 \mathrm{Aa}$ & $103.28 \mathrm{Aa}$ \\
\hline Pera de Ibotirama & 1.17 Ad & $21.96 \mathrm{Ad}$ & $31.32 \mathrm{Ad}$ & $18.34 \mathrm{Ad}$ \\
\hline Pera Olímpia & $72.44 \mathrm{Aa}$ & $84.86 \mathrm{Ab}$ & $101.11 \mathrm{Aa}$ & $82.93 \mathrm{Aa}$ \\
\hline Pera Vacinada & $60.01 \mathrm{Ab}$ & $53.07 \mathrm{Ac}$ & $71.32 \mathrm{Ab}$ & $91.66 \mathrm{Aa}$ \\
\hline Pineapple & $5.07 \mathrm{Bd}$ & $47.51 \mathrm{Ac}$ & $79.52 \mathrm{Ab}$ & $52.16 \mathrm{Ab}$ \\
\hline Russas P.S. & $5.71 \mathrm{Bd}$ & $29.48 \mathrm{Bd}$ & $62.77 \mathrm{Ab}$ & $25.01 \mathrm{Bd}$ \\
\hline Salustiana & $0.99 \mathrm{Bd}$ & $21.82 \mathrm{Ad}$ & $38.19 \mathrm{Ac}$ & $4.55 \mathrm{Bd}$ \\
\hline Seleta de Itaboraí & $10.42 \mathrm{Bd}$ & $49.65 \mathrm{Ac}$ & $59.91 \mathrm{Ac}$ & $42.10 \mathrm{Ac}$ \\
\hline Sincorá & $73.20 \mathrm{Ba}$ & $115.64 \mathrm{Aa}$ & $93.41 \mathrm{Ba}$ & $111.10 \mathrm{Aa}$ \\
\hline Valencia Chapman & $51.53 \mathrm{Ab}$ & $59.99 \mathrm{Ac}$ & $47.69 \mathrm{Ac}$ & $38.43 \mathrm{Ac}$ \\
\hline Valencia CNPMF & $10.15 \mathrm{Bd}$ & $45.13 \mathrm{Ac}$ & $43.75 \mathrm{Ac}$ & $21.36 \mathrm{Bd}$ \\
\hline Valencia CNPMF 01 & $5.27 \mathrm{Ad}$ & $13.07 \mathrm{Ad}$ & $13.45 \mathrm{Ae}$ & $16.31 \mathrm{Ad}$ \\
\hline Valencia CNPMF 02 & $9.48 \mathrm{Bd}$ & $37.30 \mathrm{Ad}$ & $53.98 \mathrm{Ac}$ & $27.46 \mathrm{Bd}$ \\
\hline Valencia CNPMF 03 & $4.14 \mathrm{Ad}$ & $29.75 \mathrm{Ad}$ & $30.97 \mathrm{Ad}$ & $17.61 \mathrm{Ad}$ \\
\hline Valencia CNPMF 21 & $23.67 \mathrm{Bc}$ & $34.84 \mathrm{Bd}$ & $39.16 \mathrm{Bc}$ & $83.75 \mathrm{Aa}$ \\
\hline Valencia CNPMF 27 & $9.29 \mathrm{Bd}$ & $39.82 \mathrm{Ac}$ & $44.66 \mathrm{Ac}$ & $32.71 \mathrm{Ac}$ \\
\hline Valencia CNPMF 36 & $15.21 \mathrm{Bd}$ & $41.91 \mathrm{Ac}$ & $54.43 \mathrm{Ac}$ & $27.31 \mathrm{Bd}$ \\
\hline Valencia CNPMF F-11 & $18.24 \mathrm{Bc}$ & $41.18 \mathrm{Ac}$ & $59.00 \mathrm{Ac}$ & $23.54 \mathrm{Bd}$ \\
\hline Valencia Criola & $4.84 \mathrm{Bd}$ & $43.23 \mathrm{Ac}$ & $36.41 \mathrm{Ad}$ & $24.68 \mathrm{Ad}$ \\
\hline Valencia Delta & $14.36 \mathrm{Ad}$ & $45.37 \mathrm{Ac}$ & $35.31 \mathrm{Ad}$ & $21.02 \mathrm{Ad}$ \\
\hline Valencia L. Shaffey & $6.27 \mathrm{Bd}$ & $20.24 \mathrm{Bd}$ & $47.33 \mathrm{Ac}$ & $20.75 \mathrm{Bd}$ \\
\hline Valencia L. White & $35.12 \mathrm{Bc}$ & $49.40 \mathrm{Ac}$ & $52.25 \mathrm{Ac}$ & 17.37 Bd \\
\hline Valencia Late & $45.81 \mathrm{Ab}$ & $13.79 \mathrm{Ad}$ & $34.19 \mathrm{Ad}$ & $22.39 \mathrm{Ad}$ \\
\hline Valencia Midknight & $6.37 \mathrm{Ad}$ & $17.15 \mathrm{Ad}$ & $19.92 \mathrm{Ae}$ & $11.16 \mathrm{Ad}$ \\
\hline Valencia Montemorelos & $26.98 \mathrm{Bc}$ & $50.40 \mathrm{Ac}$ & $67.25 \mathrm{Ab}$ & $44.16 \mathrm{Ac}$ \\
\hline Valencia Registro & $30.63 \mathrm{Bc}$ & $29.51 \mathrm{Bd}$ & $78.10 \mathrm{Ab}$ & $54.62 \mathrm{Ab}$ \\
\hline Valencia Tuxpan & $5.95 \mathrm{Ad}$ & $9.76 \mathrm{Ad}$ & $27.26 \mathrm{Ad}$ & $37.32 \mathrm{Ac}$ \\
\hline Westin & $2.74 \mathrm{Bd}$ & $43.36 \mathrm{Ac}$ & $35.01 \mathrm{Ad}$ & $27.06 \mathrm{Ad}$ \\
\hline
\end{tabular}

Means followed by the same uppercase letter in the row and lowercase in the column do not differ by the Scott-Knott mean cluster test at $5 \%$ probability. 
Table 4. Productive efficiency ( $\mathrm{kg}$ of fruits. $\mathrm{m}^{-3}$ of scion) per plant of $228 \mathrm{scion} /$ rootstock combinations comprising 57 sweet orange varieties [Citrus sinensis (L.) Osbeck] on Sunki Tropical mandarin rootstocks (TST) [Citrus sunki (Hayata) hort. ex Tanaka] and 'San Diego' (SD), 'Riverside' (RIV) and 'Indio' (IND) citrandarins [C. sunki $\times$ Poncirus trifoliata (L.) Raf.].

\begin{tabular}{|c|c|c|c|c|}
\hline \multirow{2}{*}{ Scion variety } & \multicolumn{4}{|c|}{ Rootstock } \\
\hline & TST & SD & RIV & IND \\
\hline Aquiri & $0.77 \mathrm{Ac}$ & $2.59 \mathrm{Ad}$ & $2.05 \mathrm{Ad}$ & $2.33 \mathrm{Ae}$ \\
\hline Berna & $0.03 \mathrm{Ac}$ & $0.91 \mathrm{Ae}$ & $1.16 \mathrm{Ae}$ & $2.13 \mathrm{Ae}$ \\
\hline Crescent sweet & $0.41 \mathrm{Bc}$ & $1.32 \mathrm{Be}$ & $3.30 \mathrm{Ad}$ & $1.31 \mathrm{Bf}$ \\
\hline Diva & $0.23 \mathrm{Bc}$ & $0.47 \mathrm{Be}$ & $5.41 \mathrm{Ac}$ & $0.59 \mathrm{Bf}$ \\
\hline Early Oblong & $0.53 \mathrm{Bc}$ & $1.14 \mathrm{Be}$ & $5.13 \mathrm{Ac}$ & $1.81 \mathrm{Bf}$ \\
\hline F-Menuda & $0.01 \mathrm{Ac}$ & $0.05 \mathrm{Ae}$ & $0.25 \mathrm{Ae}$ & $0.36 \mathrm{Af}$ \\
\hline Flor de Brumadinho & $0.92 \mathrm{Ac}$ & $1.74 \mathrm{Ae}$ & $1.86 \mathrm{Ae}$ & $1.76 \mathrm{Af}$ \\
\hline Hamlin CNPMF 20 & $1.92 \mathrm{Cc}$ & 4.10 Bd & $5.95 \mathrm{Ab}$ & $3.99 \mathrm{Bd}$ \\
\hline Jaffa & $0.22 \mathrm{Bc}$ & $0.48 \mathrm{Be}$ & $2.71 \mathrm{Ad}$ & $2.68 \mathrm{Ae}$ \\
\hline Mel Rosa & $0.94 \mathrm{Bc}$ & $2.99 \mathrm{Ad}$ & $3.22 \mathrm{Ad}$ & $2.89 \mathrm{Ae}$ \\
\hline Natal CNPMF 01 & $2.95 \mathrm{Bb}$ & $3.93 \mathrm{Bd}$ & $5.27 \mathrm{Ac}$ & $3.75 \mathrm{Bd}$ \\
\hline Natal CNPMF 02 & $1.27 \mathrm{Bc}$ & $3.55 \mathrm{Ad}$ & 4.72 Ac & $4.25 \mathrm{Ad}$ \\
\hline Natal CNPMF 112 & $1.54 \mathrm{Bc}$ & $1.77 \mathrm{Be}$ & $4.44 \mathrm{Ac}$ & $4.79 \mathrm{Ab}$ \\
\hline Natal Folha Murcha & $0.06 \mathrm{Ac}$ & $1.27 \mathrm{Ae}$ & $0.12 \mathrm{Ae}$ & $0.40 \mathrm{Af}$ \\
\hline Natal Ipeal & $2.93 \mathrm{Db}$ & $4.74 \mathrm{Cc}$ & $6.16 \mathrm{Bb}$ & $8.45 \mathrm{Aa}$ \\
\hline Pera Bianchi & $3.11 \mathrm{Bb}$ & $5.48 \mathrm{Ac}$ & $6.16 \mathrm{Ab}$ & $5.54 \mathrm{Ac}$ \\
\hline Pera CNPMF 01 & $4.41 \mathrm{Ba}$ & $5.15 \mathrm{Bc}$ & $6.59 \mathrm{Ab}$ & $6.45 \mathrm{Ac}$ \\
\hline Pera CNPMF 02 & $2.14 \mathrm{Cb}$ & $9.29 \mathrm{Aa}$ & 4.22 Bc & $4.57 \mathrm{Bd}$ \\
\hline Pera CNPMF A-15 & $2.18 \mathrm{Cb}$ & $10.88 \mathrm{Aa}$ & $4.01 \mathrm{Bc}$ & $5.04 \mathrm{Bd}$ \\
\hline Pera CNPMF B-12 & $4.00 \mathrm{Ba}$ & $3.01 \mathrm{Bd}$ & $5.42 \mathrm{Ac}$ & $4.36 \mathrm{Bd}$ \\
\hline Pera CNPMF C-21 & $2.65 \mathrm{Bb}$ & 4.72 Ac & $4.46 \mathrm{Ac}$ & $4.92 \mathrm{Ad}$ \\
\hline Pera CNPMF C-32 & $1.45 \mathrm{Bc}$ & $1.97 \mathrm{Be}$ & $4.04 \mathrm{Ac}$ & $3.25 \mathrm{Ae}$ \\
\hline Pera CNPMF D-3 & $5.21 \mathrm{Ba}$ & $5.68 \mathrm{Bc}$ & $7.35 \mathrm{Aa}$ & 4.70 Bd \\
\hline Pera CNPMF D-6 & $3.68 \mathrm{Aa}$ & $3.77 \mathrm{Ad}$ & $3.97 \mathrm{Ac}$ & $3.94 \mathrm{Ad}$ \\
\hline Pera CNPMF D-9 & $3.65 \mathrm{Ba}$ & $6.62 \mathrm{Ab}$ & $5.51 \mathrm{Ac}$ & $5.98 \mathrm{Ac}$ \\
\hline Pera CNPMF D-12 & $3.22 \mathrm{Bb}$ & $1.84 \mathrm{Be}$ & $5.17 \mathrm{Ac}$ & $5.50 \mathrm{Ac}$ \\
\hline Pera CNPMF D-25 & $3.28 \mathrm{Bb}$ & $3.86 \mathrm{Bd}$ & 4.79 Ac & $6.18 \mathrm{Ac}$ \\
\hline Pera CNPMF E-3 & $2.72 \mathrm{Bb}$ & $5.37 \mathrm{Ac}$ & $5.08 \mathrm{Ac}$ & $5.41 \mathrm{Ac}$ \\
\hline Pera CNPMF E-6 & $5.14 \mathrm{Ba}$ & $7.19 \mathrm{Ab}$ & $4.33 \mathrm{Bc}$ & $8.65 \mathrm{Aa}$ \\
\hline Pera GE-03 & $3.72 \mathrm{Ca}$ & $7.24 \mathrm{Bb}$ & $3.87 \mathrm{Cc}$ & $9.71 \mathrm{Aa}$ \\
\hline Pera de Ibotirama & $0.03 \mathrm{Ac}$ & $0.89 \mathrm{Ae}$ & $1.29 \mathrm{Ae}$ & $0.92 \mathrm{Af}$ \\
\hline Pera Olímpia & $4.05 \mathrm{Ba}$ & $9.23 \mathrm{Aa}$ & $8.56 \mathrm{Aa}$ & $9.23 \mathrm{Aa}$ \\
\hline Pera Vacinada & $3.19 \mathrm{Cb}$ & $5.85 \mathrm{Bc}$ & $5.56 \mathrm{Bc}$ & $7.20 \mathrm{Ab}$ \\
\hline Pineapple & $0.22 \mathrm{Bc}$ & $3.65 \mathrm{Ad}$ & $4.27 \mathrm{Ac}$ & $3.20 \mathrm{Ae}$ \\
\hline Russas P.S. & $0.23 \mathrm{Bc}$ & $1.76 \mathrm{Ae}$ & $3.05 \mathrm{Ad}$ & $1.53 \mathrm{Af}$ \\
\hline Salustiana & $0.04 \mathrm{Ac}$ & $1.14 \mathrm{Ae}$ & $1.42 \mathrm{Ae}$ & $0.22 \mathrm{Af}$ \\
\hline Seleta de Itaboraí & $0.36 \mathrm{Cc}$ & $4.65 \mathrm{Ac}$ & $3.48 \mathrm{Bd}$ & $2.70 \mathrm{Be}$ \\
\hline Sincorá & $2.81 \mathrm{Bb}$ & $6.15 \mathrm{Ab}$ & $4.29 \mathrm{Bc}$ & $5.83 \mathrm{Ac}$ \\
\hline Valencia Chapman & $2.15 \mathrm{Ab}$ & $3.72 \mathrm{Ad}$ & $2.60 \mathrm{Ad}$ & $1.92 \mathrm{Af}$ \\
\hline Valencia CNPMF & $0.47 \mathrm{Bc}$ & $2.50 \mathrm{Ad}$ & $2.21 \mathrm{Ad}$ & $0.83 \mathrm{Bf}$ \\
\hline Valencia CNPMF 01 & $0.40 \mathrm{Ac}$ & $0.82 \mathrm{Ae}$ & $0.58 \mathrm{Ae}$ & $0.92 \mathrm{Af}$ \\
\hline Valencia CNPMF 02 & $0.31 \mathrm{Ac}$ & $1.94 \mathrm{Ae}$ & $2.13 \mathrm{Ad}$ & $1.17 \mathrm{Af}$ \\
\hline Valencia CNPMF 03 & $0.15 \mathrm{Ac}$ & $1.44 \mathrm{Ae}$ & $2.38 \mathrm{Ad}$ & $0.98 \mathrm{Af}$ \\
\hline Valencia CNPMF 21 & $0.89 \mathrm{Bc}$ & $1.75 \mathrm{Be}$ & $1.61 \mathrm{Be}$ & $4.08 \mathrm{Ad}$ \\
\hline Valencia CNPMF 27 & $0.38 \mathrm{Bc}$ & $1.87 \mathrm{Ae}$ & $2.60 \mathrm{Ad}$ & $1.43 \mathrm{Af}$ \\
\hline Valencia CNPMF 36 & $0.61 \mathrm{Bc}$ & $1.92 \mathrm{Be}$ & $3.85 \mathrm{Ac}$ & $1.61 \mathrm{Bf}$ \\
\hline Valencia CNPMF F-11 & $0.66 \mathrm{Bc}$ & $2.28 \mathrm{Ad}$ & $2.78 \mathrm{Ad}$ & $1.07 \mathrm{Bf}$ \\
\hline Valencia Criola & $0.19 \mathrm{Bc}$ & $2.76 \mathrm{Ad}$ & $1.82 \mathrm{Ae}$ & $1.03 \mathrm{Bf}$ \\
\hline Valencia Delta & $0.52 \mathrm{Bc}$ & $2.61 \mathrm{Ad}$ & $2.08 \mathrm{Ad}$ & $1.12 \mathrm{Bf}$ \\
\hline Valencia L. Shaffey & $0.19 \mathrm{Ac}$ & $1.09 \mathrm{Ae}$ & $2.01 \mathrm{Ad}$ & $0.95 \mathrm{Af}$ \\
\hline Valencia L. White & $1.28 \mathrm{Bc}$ & $2.49 \mathrm{Ad}$ & $2.70 \mathrm{Ad}$ & $0.78 \mathrm{Bf}$ \\
\hline Valencia Late & $1.60 \mathrm{Ac}$ & $0.69 \mathrm{Ae}$ & $1.32 \mathrm{Ae}$ & $1.04 \mathrm{Af}$ \\
\hline Valencia Midknight & $0.41 \mathrm{Ac}$ & $1.19 \mathrm{Ae}$ & $1.63 \mathrm{Ae}$ & $1.47 \mathrm{Af}$ \\
\hline Valencia Montemorelos & $0.96 \mathrm{Cc}$ & $2.48 \mathrm{Bd}$ & 4.26 Ac & $2.65 \mathrm{Be}$ \\
\hline Valencia Registro & $1.26 \mathrm{Bc}$ & $1.49 \mathrm{Be}$ & 4.19 Ac & $3.58 \mathrm{Ae}$ \\
\hline Valencia Tuxpan & $0.23 \mathrm{Ac}$ & $0.63 \mathrm{Ae}$ & $1.46 \mathrm{Ae}$ & $1.70 \mathrm{Af}$ \\
\hline Westin & $0.14 \mathrm{Bc}$ & $2.55 \mathrm{Ad}$ & $1.78 \mathrm{Ae}$ & $1.40 \mathrm{Af}$ \\
\hline
\end{tabular}

Means followed by the same uppercase letter in the row and lowercase in the column do not differ by the Scott-Knott mean cluster test at $5 \%$ probability. 


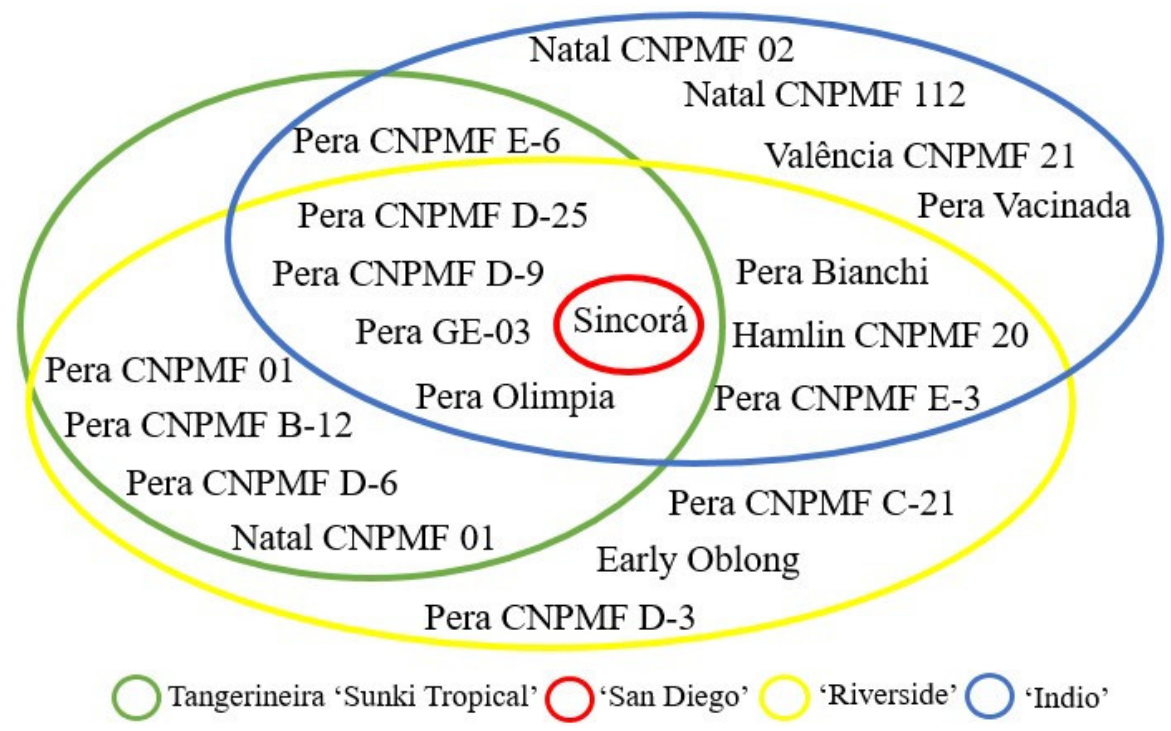

Figure 1. Orange trees that obtained higher fruit masses (kg/plant) according to the Scott-Knott test of means, among the 228 scion/rootstock combinations comprising 57 sweet orange varieties [Citrus sinensis (L.) Osbeck] on 'Sunki Tropical' (TST) mandarin rootstocks [Citrus sunki (Hayata) hort. ex Tanaka] and 'San Diego' (SD), 'Riverside' (RIV) and 'Indio' (IND) citrandarins [C. sunki $\times$ Poncirus trifoliata (L.) Raf.].

High productive efficiencies allow for increases in productivity per cultivated area (Lima et al., 2014). Rootstocks inducing high fruit productive efficiency in association with reductions in scion size are suitable for the use of high planting densities, enabling substantial reductions in the planted area without reducing fruit production, which is relevant for large and small producers who want higher yields in their crops. According to Blumer (2005), scion/rootstock combinations with these characteristics are advantageous, since, according to Carvalho et al. (2005), the productive scenario of modern citriculture foresees the use of dense planting spacing, which facilitates harvesting, pesticide application and cultural treatments in general. Santos et al. (2016) corroborate this opinion. On the other hand, as described by França et al. (2016), low productive efficiency may be associated, in some cases, with excess vegetative growth and not with lower fruit production per plant.

Regarding the harvest time, no influence of rootstocks on scions was observed. It was observed that the earliest scion varieties in this study, with harvests recorded from April to June, were 'Sincorá', 'Early Oblong', 'Salustiana', 'Westin', 'Crescent sweet' and 'Flor de Brumadinho'. The latest scion varieties were 'Hamlin CNPMF-20', 'Melrosa' and all 'Natal' and 'Valencia' selections, which had fruits harvested in August. 'Berna', 'Jaffa', 'F-Menuda', 'Aquiri', 'Russas', 'Seleta de Itaboraí' and 'Pineapple' scions, as well as 'Pera' selections, concentrated ripe fruits in the month of June. Due to the large number of combinations, fruit maturation curve analysis was not performed; however, after determining the best scion/rootstock combinations in relation to production and productive efficiency, this analysis will be performed.
Producers who want to scale their production must pay attention not only to production characteristics, but also choose scion/rootstock combinations that meet their planning and quality standards established by the consumer market, whether for fresh fruits or orange juice.

Fruit quality variations were observed, both in relation to scion cultivars and rootstocks, as well as regarding the interaction between them (Table 5). Several environmental factors and cultivation practices can influence the quality of citrus fruits. The relationship between scion and rootstock is among the most important, whose interaction has implications for the absorption of water and nutrients and hormonal effects, with direct influence on juice quality (LIU et al., 2015; LADO et al., al., 2018).

The largest cross-sectional fruit diameters (DT) were observed in 'F-Menuda', 'Seleta de Itaboraí' and 'Melrosa' scion cultivars on 'Sunki Tropical' mandarin and in 'Seleta de Itaboraí', 'Valencia Midknight' and 'Melrosa' scion cultivars on 'San Diego' citrandarin (Table 6). Regarding 'Riverside' citrandarin, the highest values for this variable were found for 'Aquiri', 'Seleta de Itaboraí', 'Diva', 'Valencia Midknight', 'Valencia L. White' and 'Melrosa' cultivars. 'Indio' citrandarin induced higher values in 'Aquiri', 'Pera de Ibotirama', 'Diva', 'Natal Folha Murcha', 'Valencia CNPMF', 'Valencia CNPMF-01', 'Valencia CNPMF-02', 'Valencia CNPMF-03', 'Valencia CNPMF-36', 'Valencia Midknight', 'Valencia Criola' and 'Valencia L. White' varieties, who showed the highest DT values. 
Table 5. Summary of the analysis of variance with sources of variation (FV), degrees of freedom (GL), mean squares of the residue (QMR) and coefficients of variation (CV) for variables cross-sectional fruit diameter (DT), soluble solids (SS), titratable acidity (TA), SS/AT ratio and juice yield (REND) of 57 sweet orange varieties [Citrus sinensis (L.) Osbeck] in combination with four rootstocks.

\begin{tabular}{ccccccc}
\hline \multirow{2}{*}{$\mathrm{FV}$} & \multirow{2}{*}{$\mathrm{GL}$} & \multicolumn{5}{c}{ QMR } \\
\cline { 3 - 6 } & & $\mathrm{DT}$ & $\mathrm{SS}$ & $\mathrm{AT}$ & $\mathrm{SS} / \mathrm{AT}$ & $\mathrm{REND}$ \\
\hline $\mathrm{PE}$ & 3 & $76,86^{* *}$ & $5,4286^{* *}$ & $1,51610^{* *}$ & $332,42^{* *}$ & $218,189^{* *}$ \\
$\mathrm{E}$ & 56 & $361,73^{* *}$ & $6,5032^{* *}$ & $0,28424^{* *}$ & $96,36^{* *}$ & $145,189^{* *}$ \\
$\mathrm{PE} \times \mathrm{E}$ & 168 & $24,88^{* *}$ & $1,1435^{* *}$ & $0,04181^{* *}$ & $9,47^{* *}$ & $13,182^{*}$ \\
$\mathrm{RES}$ & 447 & 12,38 & 0,5861 & 0,01820 & 3,68 & 10,643 \\
\hline $\mathrm{CV}(\%)$ & & 4,4 & 8,27 & 16,71 & 15,46 & 6,68 \\
\hline
\end{tabular}

${ }^{* *}$ Significant at $1 \%$ and $5 \%$ probability by the F test; ${ }^{*}$ Significant at $5 \%$ probability by the $\mathrm{F}$ test; PE $=$ Rootstock; E $=$ Graft; RES $=$ Residue.

Table 6. Cross-sectional fruit diameter $(\mathrm{mm})$ of 228 scion/rootstock combinations comprising 57 sweet orange varieties [Citrus sinensis (L.) Osbeck] on 'Sunki Tropical' (TST) mandarin rootstocks [Citrus sunki ( Hayata) hort. ex Tanaka] and 'San Diego' (SD), 'Riverside' (RIV) and 'Indio' (IND) Citrandarins [C. sunki $\times$ Poncirus trifoliata (L.) Raf.].

\begin{tabular}{lrccc}
\hline \multirow{2}{*}{ Scion variety } & \multicolumn{4}{c}{ Rootstock } \\
\cline { 2 - 5 } Aquiri & TST & SD & RIV & IND \\
Berna & $82.83 \mathrm{Ab}$ & $87.52 \mathrm{Ac}$ & $90.13 \mathrm{Aa}$ & $83.48 \mathrm{Aa}$ \\
Crescent sweet & $75.11 \mathrm{Ae}$ & 75.77 Ac & $83.14 \mathrm{Ab}$ & $79.50 \mathrm{Ab}$ \\
Diva & $85.37 \mathrm{Ab}$ & $90.08 \mathrm{Ab}$ & $86.87 \mathrm{Aa}$ & $90.49 \mathrm{Aa}$ \\
Early Oblong & $78.91 \mathrm{Ad}$ & $79.19 \mathrm{Ad}$ & $79.40 \mathrm{Ac}$ & $74.27 \mathrm{Ac}$ \\
F-Menuda & $98.83 \mathrm{Aa}$ & $84.93 \mathrm{Bc}$ & $85.15 \mathrm{Bb}$ & $80.54 \mathrm{Bb}$ \\
Flor de Brumadinho & $74.10 \mathrm{Ae}$ & $77.27 \mathrm{Ad}$ & $76.75 \mathrm{Ac}$ & $74.94 \mathrm{Ac}$ \\
Hamlin CNPMF 20 & $79.45 \mathrm{Ad}$ & $79.71 \mathrm{Ad}$ & $78.76 \mathrm{Ac}$ & $79.62 \mathrm{Ab}$ \\
Jaffa & $87.95 \mathrm{Ab}$ & $85.53 \mathrm{Ac}$ & $81.28 \mathrm{Ab}$ & $81.69 \mathrm{Ab}$ \\
Mel Rosa & $100.28 \mathrm{Aa}$ & $96.72 \mathrm{Aa}$ & $92.91 \mathrm{Aa}$ & $85.05 \mathrm{Ba}$ \\
Natal CNPMF 01 & $81.73 \mathrm{Ac}$ & $82.14 \mathrm{Ac}$ & $81.13 \mathrm{Ab}$ & $75.48 \mathrm{Ac}$ \\
Natal CNPMF 02 & $77.50 \mathrm{Ad}$ & $78.83 \mathrm{Ad}$ & $77.28 \mathrm{Ac}$ & $77.06 \mathrm{Ac}$ \\
Natal CNPMF 112 & $85.67 \mathrm{Ab}$ & $80.09 \mathrm{Bd}$ & $75.49 \mathrm{Bd}$ & $75.48 \mathrm{Bc}$ \\
Natal Folha Murcha & $84.89 \mathrm{Ab}$ & $81.26 \mathrm{Ac}$ & $86.07 \mathrm{Ab}$ & $83.97 \mathrm{Aa}$ \\
Natal Ipeal & $73.32 \mathrm{Ae}$ & $72.09 \mathrm{Ae}$ & $72.58 \mathrm{Ad}$ & $70.99 \mathrm{Ac}$ \\
Pera Bianchi & $75.02 \mathrm{Ae}$ & $73.51 \mathrm{Ae}$ & $76.15 \mathrm{Ac}$ & $76.43 \mathrm{Ac}$ \\
Pera CNPMF 01 & $73.70 \mathrm{Ae}$ & $74.94 \mathrm{Ad}$ & $75.67 \mathrm{Ad}$ & $77.56 \mathrm{Ac}$ \\
Pera CNPMF 02 & $73.36 \mathrm{Ae}$ & $72.33 \mathrm{Ae}$ & $74.62 \mathrm{Ad}$ & $71.71 \mathrm{Ac}$ \\
Pera CNPMF A-15 & $76.36 \mathrm{Ad}$ & $68.06 \mathrm{Be}$ & $78.77 \mathrm{Ac}$ & $77.66 \mathrm{Ac}$ \\
Pera CNPMF B-12 & $72.44 \mathrm{Ae}$ & $69.79 \mathrm{Ae}$ & $75.50 \mathrm{Ad}$ & $73.71 \mathrm{Ac}$ \\
Pera CNPMF C-21 & $74.34 \mathrm{Ae}$ & $70.72 \mathrm{Ae}$ & $71.45 \mathrm{Ad}$ & $72.56 \mathrm{Ac}$ \\
Pera CNPMF C-32 & $76.94 \mathrm{Ad}$ & $78.02 \mathrm{Ad}$ & $80.19 \mathrm{Ac}$ & $74.40 \mathrm{Ac}$ \\
Pera CNPMF D-3 & $69.39 \mathrm{Ae}$ & $73.08 \mathrm{Ae}$ & $74.39 \mathrm{Ad}$ & $75.69 \mathrm{Ac}$ \\
Pera CNPMF D-6 & $76.66 \mathrm{Ad}$ & $79.66 \mathrm{Ad}$ & $75.22 \mathrm{Ad}$ & $80.22 \mathrm{Ab}$ \\
Pera CNPMF D-9 & $71.21 \mathrm{Ae}$ & $76.76 \mathrm{Ad}$ & $78.90 \mathrm{Ac}$ & $75.51 \mathrm{Ac}$ \\
Pera CNPMF D-12 & $71.50 \mathrm{Ae}$ & $72.39 \mathrm{Ae}$ & $71.72 \mathrm{Ad}$ & $71.38 \mathrm{Ac}$ \\
Pera CNPMF D-25 & $78.21 \mathrm{Bd}$ & $77.62 \mathrm{Bd}$ & $83.60 \mathrm{Ab}$ & $74.39 \mathrm{Bc}$ \\
Pera CNPMF E-3 & $70.50 \mathrm{Ae}$ & $70.13 \mathrm{Ae}$ & $70.30 \mathrm{Ad}$ & $70.77 \mathrm{Ac}$ \\
Pera CNPMF E-6 & $70.66 \mathrm{Ae}$ & $70.78 \mathrm{Ae}$ & $71.84 \mathrm{Ad}$ & $73.97 \mathrm{Ac}$ \\
Pera GE-03 & $75.82 \mathrm{Bd}$ & $72.23 \mathrm{Be}$ & $80.82 \mathrm{Ab}$ & $72.88 \mathrm{Bc}$ \\
\hline
\end{tabular}


continuation...

\begin{tabular}{lrlll}
\hline Pera de Ibotirama & $86.28 \mathrm{Ab}$ & $86.59 \mathrm{Ac}$ & $83.01 \mathrm{Ab}$ & $84.72 \mathrm{Aa}$ \\
Pera Olímpia & $78.21 \mathrm{Ad}$ & $72.66 \mathrm{Ae}$ & $76.44 \mathrm{Ac}$ & $79.01 \mathrm{Ab}$ \\
Pera Vacinada & $72.49 \mathrm{Ae}$ & $70.50 \mathrm{Ae}$ & $71.44 \mathrm{Ad}$ & $74.76 \mathrm{Ac}$ \\
Pineapple & $87.62 \mathrm{Ab}$ & $83.75 \mathrm{Ac}$ & $78.17 \mathrm{Bc}$ & $79.31 \mathrm{Bb}$ \\
Russas P.S. & $81.41 \mathrm{Ac}$ & $75.04 \mathrm{Ad}$ & $78.39 \mathrm{Ac}$ & $82.14 \mathrm{Ab}$ \\
Salustiana & $84.93 \mathrm{Ab}$ & $80.17 \mathrm{Ad}$ & $82.17 \mathrm{Ab}$ & $80.70 \mathrm{Ab}$ \\
Seleta de Itaboraí & $103.75 \mathrm{Aa}$ & $93.67 \mathrm{Ba}$ & $88.81 \mathrm{Ba}$ & $89.90 \mathrm{Ba}$ \\
Sincorá & $77.90 \mathrm{Ad}$ & $80.05 \mathrm{Ad}$ & $74.32 \mathrm{Bd}$ & $72.37 \mathrm{Bc}$ \\
Valencia Chapman & $80.08 \mathrm{Ad}$ & $82.17 \mathrm{Ac}$ & $77.56 \mathrm{Bc}$ & $74.01 \mathrm{Bc}$ \\
Valencia CNPMF & $82.95 \mathrm{Ac}$ & $80.84 \mathrm{Ac}$ & $84.97 \mathrm{Ab}$ & $83.87 \mathrm{Aa}$ \\
Valencia CNPMF 01 & $81.69 \mathrm{Ac}$ & $86.48 \mathrm{Ac}$ & $83.34 \mathrm{Ab}$ & $86.09 \mathrm{Aa}$ \\
Valencia CNPMF 02 & $85.52 \mathrm{Ab}$ & $85.12 \mathrm{Ac}$ & $83.24 \mathrm{Ab}$ & $84.38 \mathrm{Aa}$ \\
Valencia CNPMF 03 & $81.48 \mathrm{Ac}$ & $80.90 \mathrm{Ac}$ & $82.19 \mathrm{Ab}$ & $83.57 \mathrm{Aa}$ \\
Valencia CNPMF 21 & $84.18 \mathrm{Ac}$ & $88.77 \mathrm{Ab}$ & $81.25 \mathrm{Bb}$ & $78.52 \mathrm{Bc}$ \\
Valencia CNPMF 27 & $81.95 \mathrm{Ac}$ & $82.70 \mathrm{Ac}$ & $82.65 \mathrm{Ab}$ & $82.07 \mathrm{Ab}$ \\
Valencia CNPMF 36 & $89.15 \mathrm{Ab}$ & $89.47 \mathrm{Ab}$ & $84.43 \mathrm{Ab}$ & $86.81 \mathrm{Aa}$ \\
Valencia CNPMF F-11 & $87.16 \mathrm{Ab}$ & $84.87 \mathrm{Ac}$ & $78.43 \mathrm{Bc}$ & $82.93 \mathrm{Ab}$ \\
Valencia Criola & $83.59 \mathrm{Ac}$ & $76.61 \mathrm{Bd}$ & $79.49 \mathrm{Bc}$ & $83.85 \mathrm{Aa}$ \\
Valencia Delta & $83.47 \mathrm{Ac}$ & $85.76 \mathrm{Ac}$ & $81.60 \mathrm{Ab}$ & $81.23 \mathrm{Ab}$ \\
Valencia L. Shaffey & $81.22 \mathrm{Ac}$ & $83.40 \mathrm{Ac}$ & $78.41 \mathrm{Ac}$ & $80.92 \mathrm{Ab}$ \\
Valencia L. White & $83.36 \mathrm{Ac}$ & $88.33 \mathrm{Ab}$ & $89.95 \mathrm{Aa}$ & $84.14 \mathrm{Aa}$ \\
Valencia Late & $81.44 \mathrm{Ac}$ & $81.49 \mathrm{Ac}$ & $81.61 \mathrm{Ab}$ & $81.92 \mathrm{Ab}$ \\
Valencia Midknight & $91.70 \mathrm{Ab}$ & $95.70 \mathrm{Aa}$ & $92.06 \mathrm{Aa}$ & $88.67 \mathrm{Aa}$ \\
Valencia Montemorelos & $82.16 \mathrm{Ac}$ & $84.29 \mathrm{Ac}$ & $84.67 \mathrm{Ab}$ & $82.50 \mathrm{Ab}$ \\
Valencia Registro & $79.64 \mathrm{Bd}$ & $86.03 \mathrm{Ac}$ & $75.31 \mathrm{Bd}$ & $79.41 \mathrm{Bb}$ \\
Valencia Tuxpan & $80.61 \mathrm{Ac}$ & $87.07 \mathrm{Ac}$ & $81.62 \mathrm{Ab}$ & $79.92 \mathrm{Ab}$ \\
Westin & $84.67 \mathrm{Ab}$ & $79.80 \mathrm{Bd}$ & $79.54 \mathrm{Bc}$ & $76.47 \mathrm{Bc}$ \\
\hline
\end{tabular}

Means followed by the same uppercase letter in the row and lowercase in the column do not differ by the Scott-Knott mean cluster test at $5 \%$ probability.

The overall mean for the 26 combinations with the highest DT values was $89.27 \mathrm{~mm}$. 'Seleta de Itaboraí' and 'Melrosa' cultivars presented higher DT values in the four rootstocks. However, according to the Ceagesp classification (2011), regarding the class of sweet and low-acidity oranges, it was observed that more than $94 \%$ of combinations evaluated in this study fall into class of 'large' fruits, with DT above $71 \mathrm{~mm}$, rated ' $A$ ' and the others rated 'B', with 'intermediate' size, between 65 and $71 \mathrm{~mm}$.

'Indio' citrandarin, in 53\% of combinations, was the rootstock that induced the highest average DT value. According to Liu et al. (2015), the diameter of citrus fruits is related to the rootstock used, as it influences plant metabolism, regulating hormonal interactions and cell size. Therefore, in cases where the aim is production to supply the fresh fruit market, both scion variety and rootstock variety must be properly defined for the orchard implantation.
Differences were observed among combinations regarding the soluble solids (SS) content of fruits. Among combinations, $22 \%$ had fruits with values equal to or greater than $10{ }^{\circ} \mathrm{Brix}$, which, according to the Ceagesp classification (2011), qualifies them for consumption as good quality fresh fruits (Table 7). 
Table 7. Soluble solids ( ${ }^{\circ}$ Brix) of 228 scion/rootstock combinations comprising 57 sweet orange varieties [Citrus sinensis (L.) Osbeck] on 'Tropical Sunki' (TST) mandarin rootstocks [Citrus sunki (Hayata) hort. ex Tanaka] and 'San Diego' (SD), 'Riverside' (RIV) and 'Indio' (IND) citrandarins [C. sunki $\times$ Poncirus trifoliata (L.) Raf.].

\begin{tabular}{|c|c|c|c|c|}
\hline \multirow{2}{*}{ Scion variety } & \multicolumn{4}{|c|}{ Rootstock } \\
\hline & TST & SD & RIV & IND \\
\hline Aquiri & $8.77 \mathrm{Ac}$ & $8.70 \mathrm{Ab}$ & $8.23 \mathrm{Ac}$ & $8.73 \mathrm{~A}$ \\
\hline Berna & $8.50 \mathrm{Ac}$ & $8.23 \mathrm{Ab}$ & $8.33 \mathrm{Ac}$ & $8.50 \mathrm{~A}$ \\
\hline Crescent sweet & $10.47 \mathrm{Aa}$ & $10.50 \mathrm{Aa}$ & $10.07 \mathrm{Ab}$ & $10.27 A$ \\
\hline Diva & $8.90 \mathrm{Ac}$ & $7.87 \mathrm{Ab}$ & $8.60 \mathrm{Ac}$ & $8.63 \mathrm{~A}$ \\
\hline Early Oblong & $8.57 \mathrm{Ac}$ & $9.53 \mathrm{Ab}$ & $9.17 \mathrm{Ac}$ & $10.20 \mathrm{~A}$ \\
\hline F-Menuda & $10.90 \mathrm{Aa}$ & $9.37 \mathrm{Ab}$ & $10.03 \mathrm{Ab}$ & $8.67 \mathrm{~A}$ \\
\hline Flor de Brumadinho & $9.43 \mathrm{Ab}$ & $8.50 \mathrm{Ab}$ & $8.93 \mathrm{Ac}$ & $8.77 \mathrm{~A}$ \\
\hline Hamlin CNPMF 20 & $9.23 \mathrm{Ac}$ & $8.93 \mathrm{Ab}$ & $9.00 \mathrm{Ac}$ & $8.60 \mathrm{~A}$ \\
\hline Jaffa & $9.30 \mathrm{Ac}$ & $9.57 \mathrm{Ab}$ & $9.23 \mathrm{Ac}$ & $9.53 \mathrm{~A}$ \\
\hline Mel Rosa & $9.10 \mathrm{Ac}$ & $9.07 \mathrm{Ab}$ & $9.87 \mathrm{Ab}$ & $10.17 \mathrm{~A}$ \\
\hline Natal CNPMF 01 & $8.23 \mathrm{Ac}$ & $8.37 \mathrm{Ab}$ & $9.03 \mathrm{Ac}$ & $9.03 \mathrm{~A}$ \\
\hline Natal CNPMF 02 & $9.60 \mathrm{Ab}$ & $9.43 \mathrm{Ab}$ & $10.07 \mathrm{Ab}$ & $9.13 \mathrm{~A}$ \\
\hline Natal CNPMF 112 & $7.50 \mathrm{Bc}$ & $9.27 \mathrm{Ab}$ & 8.77 Ac & $8.87 \mathrm{~A}$ \\
\hline Natal Folha Murcha & $8.17 \mathrm{Ac}$ & $9.00 \mathrm{Ab}$ & $8.53 \mathrm{Ac}$ & $8.83 \mathrm{~A}$ \\
\hline Natal Ipeal & $10.30 \mathrm{Aa}$ & $10.80 \mathrm{Aa}$ & $10.60 \mathrm{Aa}$ & $9.71 \mathrm{~A}$ \\
\hline Pera Bianchi & $10.43 \mathrm{Aa}$ & $10.60 \mathrm{Aa}$ & $9.53 \mathrm{Bc}$ & $9.07 \mathrm{E}$ \\
\hline Pera CNPMF 01 & $10.30 \mathrm{Aa}$ & $9.95 \mathrm{Aa}$ & $9.63 \mathrm{Ab}$ & $8.77 \mathrm{~A}$ \\
\hline Pera CNPMF 02 & $10.37 \mathrm{Aa}$ & $10.30 \mathrm{Aa}$ & $10.10 \mathrm{Aa}$ & $9.57 \mathrm{~A}$ \\
\hline Pera CNPMF A-15 & $9.90 \mathrm{Bb}$ & $11.63 \mathrm{Aa}$ & $9.70 \mathrm{Bb}$ & $10.07 \mathrm{~B}$ \\
\hline Pera CNPMF B-12 & $10.37 \mathrm{Aa}$ & $10.96 \mathrm{Aa}$ & $9.87 \mathrm{Ab}$ & $9.43 A$ \\
\hline Pera CNPMF C-21 & $9.43 \mathrm{Bb}$ & $11.20 \mathrm{Aa}$ & $10.90 \mathrm{Aa}$ & $10.53 \mathrm{~A}$ \\
\hline Pera CNPMF C-32 & $9.73 \mathrm{Ab}$ & $10.00 \mathrm{Aa}$ & $10.20 \mathrm{Ab}$ & $9.03 \mathrm{~A}$ \\
\hline Pera CNPMF D-3 & $11.67 \mathrm{Aa}$ & $11.37 \mathrm{Aa}$ & $10.60 \mathrm{Aa}$ & $8.77 \mathrm{~B}$ \\
\hline Pera CNPMF D-6 & $9.73 \mathrm{Ab}$ & $10.50 \mathrm{Aa}$ & $10.17 \mathrm{Ab}$ & $9.23 \mathrm{~A}$ \\
\hline Pera CNPMF D-9 & $9.93 \mathrm{Ab}$ & $10.23 \mathrm{Aa}$ & $8.63 \mathrm{Bc}$ & $10.30 \mathrm{~A}$ \\
\hline Pera CNPMF D-12 & $11.13 \mathrm{Aa}$ & $11.57 \mathrm{Aa}$ & $11.03 \mathrm{Aa}$ & $10.73 \mathrm{~A}$ \\
\hline Pera CNPMF D-25 & $8.70 \mathrm{Bc}$ & $10.60 \mathrm{Aa}$ & $8.67 \mathrm{Bc}$ & $9.43 \mathrm{~B}$ \\
\hline Pera CNPMF E-3 & $9.33 \mathrm{Bc}$ & $11.27 \mathrm{Aa}$ & $10.30 \mathrm{Ab}$ & $9.17 \mathrm{~B}$ \\
\hline Pera CNPMF E-6 & $9.10 \mathrm{Bc}$ & $11.00 \mathrm{Aa}$ & $9.90 \mathrm{Ab}$ & $8.67 \mathrm{~B}$ \\
\hline Pera GE-03 & $9.77 \mathrm{Bb}$ & $11.57 \mathrm{Aa}$ & $8.67 \mathrm{Bc}$ & $9.50 \mathrm{~B}$ \\
\hline Pera de Ibotiras & $8.30 \mathrm{Ac}$ & $8.37 \mathrm{Ab}$ & 7.70 Ac & $8.00 \mathrm{~A}$ \\
\hline Pera Olímpia & $9.80 \mathrm{Ab}$ & $10.73 \mathrm{Aa}$ & $8.90 \mathrm{Bc}$ & $8.57 \mathrm{~B}$ \\
\hline Pera Vacinada & $9.97 \mathrm{Bb}$ & $12.07 \mathrm{Aa}$ & $10.90 \mathrm{Aa}$ & $9.30 \mathrm{~B}$ \\
\hline Pineapple & $8.87 \mathrm{Ac}$ & $8.77 \mathrm{Ab}$ & $9.20 \mathrm{Ac}$ & $8.93 \mathrm{~A}$ \\
\hline Russas P.S. & $9.20 \mathrm{Ac}$ & $8.97 \mathrm{Ab}$ & $8.60 \mathrm{Ac}$ & $8.73 \mathrm{~A}$ \\
\hline Salustiana & $9.90 \mathrm{Ab}$ & $9.03 \mathrm{Ab}$ & $8.87 \mathrm{Ac}$ & $9.60 \mathrm{~A}$ \\
\hline Seleta de Itaboraí & $8.43 \mathrm{Bc}$ & $10.65 \mathrm{Aa}$ & $11.63 \mathrm{Aa}$ & $10.27 \mathrm{~A}$ \\
\hline Sincorá & $9.63 \mathrm{Ab}$ & $9.60 \mathrm{Ab}$ & $9.40 \mathrm{Ac}$ & $9.47 \mathrm{~A}$ \\
\hline Valencia Chapman & $8.27 \mathrm{Ac}$ & $8.10 \mathrm{Ab}$ & $9.57 \mathrm{Ab}$ & $9.07 \mathrm{~A}$ \\
\hline Valencia CNPMF & $8.60 \mathrm{Ac}$ & $9.23 \mathrm{Ab}$ & 8.27 Ac & $8.83 \mathrm{~A}$ \\
\hline Valencia CNPMF 01 & $8.27 \mathrm{Ac}$ & $8.07 \mathrm{Ab}$ & $8.60 \mathrm{Ac}$ & $8.13 \mathrm{~A}$ \\
\hline Valencia CNPMF 02 & $8.37 \mathrm{Ac}$ & $8.97 \mathrm{Ab}$ & $9.07 \mathrm{Ac}$ & $7.87 \mathrm{~A}$ \\
\hline Valencia CNPMF 03 & $9.37 \mathrm{Ac}$ & $8.07 \mathrm{Ab}$ & $7.93 \mathrm{Ac}$ & $8.50 \mathrm{~A}$ \\
\hline Valencia CNPMF 21 & $9.00 \mathrm{Ac}$ & $8.00 \mathrm{Ab}$ & $9.20 \mathrm{Ac}$ & 8.70 \\
\hline
\end{tabular}


continuation..

\begin{tabular}{lllll}
\hline Valencia CNPMF 27 & $7.90 \mathrm{Ac}$ & $8.57 \mathrm{Ab}$ & $8.40 \mathrm{Ac}$ & $8.40 \mathrm{Ab}$ \\
Valencia CNPMF 36 & $8.40 \mathrm{Ac}$ & $8.03 \mathrm{Ab}$ & $8.87 \mathrm{Ac}$ & $8.63 \mathrm{Ab}$ \\
Valencia CNPMF F-11 & $9.17 \mathrm{Ac}$ & $9.33 \mathrm{Ab}$ & $9.60 \mathrm{Ab}$ & $8.77 \mathrm{Ab}$ \\
Valencia Criola & $8.10 \mathrm{Ac}$ & $9.43 \mathrm{Ab}$ & $9.03 \mathrm{Ac}$ & $8.07 \mathrm{Ab}$ \\
Valencia Delta & $8.30 \mathrm{Ac}$ & $8.93 \mathrm{Ab}$ & $8.33 \mathrm{Ac}$ & $9.20 \mathrm{Ab}$ \\
Valencia L. Shaffey & $8.07 \mathrm{Ac}$ & $8.67 \mathrm{Ab}$ & $9.27 \mathrm{Ac}$ & $9.17 \mathrm{Ab}$ \\
Valencia L. White & $9.17 \mathrm{Ac}$ & $8.90 \mathrm{Ab}$ & $7.77 \mathrm{Bc}$ & $9.63 \mathrm{Aa}$ \\
Valencia Late & $9.67 \mathrm{Ab}$ & $8.80 \mathrm{Ab}$ & $8.67 \mathrm{Ac}$ & $9.23 \mathrm{Ab}$ \\
Valencia Midknight & $8.20 \mathrm{Ac}$ & $8.30 \mathrm{Ab}$ & $8.67 \mathrm{Ac}$ & $8.10 \mathrm{Ab}$ \\
Valencia Montemorelos & $9.10 \mathrm{Ac}$ & $8.33 \mathrm{Ab}$ & $7.70 \mathrm{Ac}$ & $9.20 \mathrm{Ab}$ \\
Valencia Registro & $9.03 \mathrm{Ac}$ & $8.13 \mathrm{Bb}$ & $9.60 \mathrm{Ab}$ & $7.57 \mathrm{Bb}$ \\
Valencia Tuxpan & $8.47 \mathrm{Ac}$ & $8.37 \mathrm{Ab}$ & $8.17 \mathrm{Ac}$ & $8.17 \mathrm{Ab}$ \\
Westin & $9.23 \mathrm{Ac}$ & $10.00 \mathrm{Aa}$ & $9.30 \mathrm{Ac}$ & $9.70 \mathrm{Aa}$ \\
\hline
\end{tabular}

Means followed by the same uppercase letter in the row and lowercase in the column do not differ by the Scott-Knott mean cluster test at $5 \%$ probability.

'Pera CNPMF-02', 'Pera CNPMF D-12' and 'Natal Ipeal' orange varieties were those with the highest SS levels on the four rootstocks under study. 'Seleta de Itaboraí' scion stood out on 'San Diego', 'Riverside' and 'Indio', while 'Crescent sweet' scion on 'Sunki Tropical', 'San Diego' and 'Indio' rootstocks. The SS content is one of the most important variables in defining fruit quality, as it significantly influences flavor (TARANTINO et al., 2018). According to Couto et al. (2018), small variations, smaller than $1^{\circ}$ Brix, in the minimum acceptable value ( 9 to 10 ) do not lead to difference in palatability for industrialization; however, this difference can be observed in fresh consumption. Thus, scion/rootstock combinations that produce fruits with higher SS values are more desirable, as they serve both the fresh fruit market and for the juice industry (MORETTO et al., 2019).

Regarding titratable acidity (AT), it was observed in Table 8 that the combinations that presented superior results were those involving 'Sunki Tropical' mandarin and 'Hamlin CNPMF-20', 'Natal CNPMF-02', 'Valencia CNPMF', 'Valencia CNPMF-27', 'Valencia Late', 'Valencia Chapman', 'Valencia L. White', Valencia Montemorelos' and 'Valencia Registro' varieties. 'Pera CNPMF A-15', 'Pera CNPMF B-12', 'Pera CNPMF C-21', 'Pera CNPMF C-32', 'Pera CNPMF D-3', 'Pera GE-03', 'F-Menuda', 'Salustiana', 'Pineapple', 'Hamlin CNPMF-20', 'Natal CNPMF-01', 'Natal CNPMF-02', 'Natal CNPMF-112', 'Natal Folha Murcha, Valencia CNPMF', 'Valencia CNPMF-27', 'Valencia CNPMF F-11', 'Valencia Criola', 'Valencia Late', 'Valencia Chapman', 'Valencia L. White', 'Valencia Montemorelo's, 'Valencia Registro' and 'Valencia CNPMF-21' provided fruits with higher acidity when combined with 'San Diego' citrandarin.
On 'Riverside' citrandarin, fruits with higher AT levels were observed in 'Pera CNPMF D-3', 'Pera Vacinada', 'Jaffa', 'Sincorá', 'Early Oblong', 'Russas', 'Salustiana', 'Pineapple', 'Hamlin CNPMF-20', 'Natal CNPMF-01', 'Natal CNPMF-02', 'Natal CNPMF-112', 'Natal Folha Murcha', 'Valência CNPMF-02', 'Valencia CNPMF', 'Valencia CNPMF-03', 'Valencia CNPMF-27', 'Valencia CNPMF F-11', 'Valencia Criola', 'Valencia Late', 'Valencia L. Shaffey', 'Valencia Chapman', 'Valencia Registro' and 'Valencia CNPMF-21' varieties. On 'Indio' citrandarin, 'Valencia CNPMF' showed high AT value. 
Table 8. Titratable acidity (\%, citric acid) of fruits from 228 scion/rootstock combinations comprising 57 sweet orange varieties [Citrus sinensis (L.) Osbeck] on 'Sunki Tropical' (TST) mandarin rootstocks [Citrus sunki (Hayata) hort. ex Tanaka] and 'San Diego' (SD), 'Riverside' (RIV) and 'Indio' (IND) citrandarins [C. sunki $\times$ Poncirus trifoliata (L.) Raf.].

\begin{tabular}{|c|c|c|c|c|}
\hline \multirow{2}{*}{ Scion variety } & \multicolumn{4}{|c|}{ Rootstock } \\
\hline & TST & SD & RIV & IND \\
\hline Aquiri & $0.64 \mathrm{Ad}$ & $0.62 \mathrm{Ab}$ & $0.68 \mathrm{Ab}$ & $0.89 \mathrm{Ac}$ \\
\hline Berna & $0.51 \mathrm{Bd}$ & $0.48 \mathrm{Bb}$ & $0.53 \mathrm{Bb}$ & $0.78 \mathrm{Ad}$ \\
\hline Crescent sweet & $0.76 \mathrm{Ad}$ & $0.65 \mathrm{Ab}$ & $0.61 \mathrm{Ab}$ & $0.66 \mathrm{Ae}$ \\
\hline Diva & $0.76 \mathrm{Ad}$ & $0.64 \mathrm{Ab}$ & $0.63 \mathrm{Ab}$ & $0.62 \mathrm{Ae}$ \\
\hline Early Oblong & $0.85 \mathrm{Ac}$ & $0.74 \mathrm{Ab}$ & $0.84 \mathrm{Aa}$ & $0.99 \mathrm{Ac}$ \\
\hline F-Menuda & $0.76 \mathrm{Ad}$ & $0.88 \mathrm{Aa}$ & $0.72 \mathrm{Ab}$ & $1.11 \mathrm{Ab}$ \\
\hline Flor de Brumadinho & $0.59 \mathrm{Ad}$ & $0.36 \mathrm{Ab}$ & $0.42 \mathrm{Ab}$ & $0.44 \mathrm{Ae}$ \\
\hline Hamlin CNPMF 20 & $1.27 \mathrm{Aa}$ & $0.96 \mathrm{Ba}$ & $0.93 \mathrm{Ba}$ & $0.88 \mathrm{Bd}$ \\
\hline Jaffa & $0.92 \mathrm{Ac}$ & $0.70 \mathrm{Bb}$ & $1.02 \mathrm{Aa}$ & $1.11 \mathrm{Ab}$ \\
\hline Mel Rosa & $0.76 \mathrm{Ad}$ & $0.44 \mathrm{Bb}$ & $0.57 \mathrm{Bb}$ & $0.82 \mathrm{Ad}$ \\
\hline Natal CNPMF 01 & $0.97 \mathrm{Ac}$ & $1.00 \mathrm{Aa}$ & $0.99 \mathrm{Aa}$ & $1.01 \mathrm{Ac}$ \\
\hline Natal CNPMF 02 & $1.27 \mathrm{Aa}$ & $0.98 \mathrm{Ba}$ & $0.87 \mathrm{Ba}$ & $1.19 \mathrm{Ab}$ \\
\hline Natal CNPMF 112 & $1.05 \mathrm{Ab}$ & $1.12 \mathrm{Aa}$ & $1.10 \mathrm{Aa}$ & $0.99 \mathrm{Ac}$ \\
\hline Natal Folha Murcha & $1.01 \mathrm{Ab}$ & $0.80 \mathrm{Aa}$ & $0.82 \mathrm{Aa}$ & $0.91 \mathrm{Ac}$ \\
\hline Natal Ipeal & $0.92 \mathrm{Ac}$ & $0.53 \mathrm{Bb}$ & $0.52 \mathrm{Bb}$ & $0.69 \mathrm{Be}$ \\
\hline Pera Bianchi & $0.72 \mathrm{Ad}$ & $0.64 \mathrm{Ab}$ & $0.63 \mathrm{Ab}$ & $0.61 \mathrm{Ae}$ \\
\hline Pera CNPMF 01 & $0.77 \mathrm{Ad}$ & $0.49 \mathrm{Ab}$ & $0.56 \mathrm{Ab}$ & $0.65 \mathrm{Ae}$ \\
\hline Pera CNPMF 02 & $0.95 \mathrm{Ac}$ & $0.68 \mathrm{Bb}$ & $0.52 \mathrm{Bb}$ & $0.55 \mathrm{Be}$ \\
\hline Pera CNPMF A-15 & $0.88 \mathrm{Ac}$ & $1.04 \mathrm{Aa}$ & $0.56 \mathrm{Bb}$ & $0.50 \mathrm{Be}$ \\
\hline Pera CNPMF B-12 & $0.67 \mathrm{Bd}$ & $0.84 \mathrm{Aa}$ & $0.59 \mathrm{Bb}$ & $0.51 \mathrm{Be}$ \\
\hline Pera CNPMF C-21 & $1.01 \mathrm{Ab}$ & $1.02 \mathrm{Aa}$ & $0.71 \mathrm{Bb}$ & $0.93 \mathrm{Ac}$ \\
\hline Pera CNPMF C-32 & $0.96 \mathrm{Ac}$ & $0.89 \mathrm{Aa}$ & $0.63 \mathrm{Bb}$ & $0.84 \mathrm{Ad}$ \\
\hline Pera CNPMF D-3 & $1.08 \mathrm{Ab}$ & $0.78 \mathrm{Ba}$ & $0.76 \mathrm{Ba}$ & $0.86 \mathrm{Bd}$ \\
\hline Pera CNPMF D-6 & $0.90 \mathrm{Ac}$ & $0.64 \mathrm{Bb}$ & $0.65 \mathrm{Bb}$ & $0.60 \mathrm{Be}$ \\
\hline Pera CNPMF D-9 & $0.95 \mathrm{Ac}$ & $0.67 \mathrm{Bb}$ & $0.61 \mathrm{Bb}$ & $0.64 \mathrm{Be}$ \\
\hline Pera CNPMF D-12 & $0.71 \mathrm{Ad}$ & $0.59 \mathrm{Ab}$ & $0.65 \mathrm{Ab}$ & $0.61 \mathrm{Ae}$ \\
\hline Pera CNPMF D-25 & $0.90 \mathrm{Ac}$ & $0.74 \mathrm{Ab}$ & $0.63 \mathrm{Ab}$ & $0.80 \mathrm{Ad}$ \\
\hline Pera CNPMF E-3 & $0.81 \mathrm{Ac}$ & $0.59 \mathrm{Bb}$ & $0.52 \mathrm{Bb}$ & $0.63 \mathrm{Be}$ \\
\hline Pera CNPMF E-6 & $0.70 \mathrm{Ad}$ & $0.63 \mathrm{Ab}$ & $0.58 \mathrm{Ab}$ & $0.61 \mathrm{Ae}$ \\
\hline Pera GE-03 & $1.04 \mathrm{Ab}$ & $0.78 \mathrm{Ba}$ & $0.57 \mathrm{Bb}$ & $0.68 \mathrm{Be}$ \\
\hline Pera de Ibotirama & $0.61 \mathrm{Ad}$ & $0.45 \mathrm{Ab}$ & $0.56 \mathrm{Ab}$ & $0.73 \mathrm{Ad}$ \\
\hline Pera Olímpia & $0.78 \mathrm{Ad}$ & $0.61 \mathrm{Ab}$ & $0.61 \mathrm{Ab}$ & $0.67 \mathrm{Ae}$ \\
\hline Pera Vacinada & $0.81 \mathrm{Ac}$ & $0.73 \mathrm{Ab}$ & $0.77 \mathrm{Aa}$ & $0.77 \mathrm{Ad}$ \\
\hline Pineapple & $1.00 \mathrm{Ab}$ & $0.91 \mathrm{Aa}$ & $0.91 \mathrm{Aa}$ & $1.09 \mathrm{Ab}$ \\
\hline Russas P.S. & $0.84 \mathrm{Ac}$ & $0.75 \mathrm{Ab}$ & $0.80 \mathrm{Aa}$ & $0.84 \mathrm{Ad}$ \\
\hline Salustiana & $0.86 \mathrm{Ac}$ & $1.06 \mathrm{Aa}$ & $0.93 \mathrm{Aa}$ & $1.06 \mathrm{Ac}$ \\
\hline Seleta de Itaboraí & $0.60 \mathrm{Ad}$ & $0.48 \mathrm{Ab}$ & $0.54 \mathrm{Ab}$ & $0.74 \mathrm{Ad}$ \\
\hline Sincorá & $0.89 \mathrm{Ac}$ & $0.75 \mathrm{Ab}$ & $0.85 \mathrm{Aa}$ & $0.76 \mathrm{Ad}$ \\
\hline Valencia Chapman & $1.30 \mathrm{Aa}$ & $0.95 \mathrm{Ba}$ & $0.93 \mathrm{Ba}$ & $1.17 \mathrm{Ab}$ \\
\hline Valencia CNPMF & $1.47 \mathrm{Aa}$ & $0.85 \mathrm{Ba}$ & $0.84 \mathrm{Ba}$ & $1.44 \mathrm{Aa}$ \\
\hline Valencia CNPMF 01 & $0.83 \mathrm{Ac}$ & $0.56 \mathrm{Bb}$ & $0.71 \mathrm{Ab}$ & $0.52 \mathrm{Be}$ \\
\hline Valencia CNPMF 02 & $1.12 \mathrm{Ab}$ & $0.69 \mathrm{Bb}$ & $0.90 \mathrm{Ba}$ & $0.78 \mathrm{Bd}$ \\
\hline Valencia CNPMF 03 & $1.15 \mathrm{Ab}$ & $0.77 \mathrm{Bb}$ & $0.81 \mathrm{Ba}$ & $0.71 \mathrm{Bd}$ \\
\hline Valencia CNPMF 21 & $1.03 \mathrm{Ab}$ & $0.82 \mathrm{Aa}$ & $0.89 \mathrm{Aa}$ & $0.98 \mathrm{Ac}$ \\
\hline
\end{tabular}


continuation...

\begin{tabular}{lllll} 
Valencia CNPMF 27 & $1.21 \mathrm{Aa}$ & $0.94 \mathrm{Ba}$ & $0.86 \mathrm{Ba}$ & $0.81 \mathrm{Bd}$ \\
Valencia CNPMF 36 & $0.99 \mathrm{Ab}$ & $0.70 \mathrm{Bb}$ & $0.72 \mathrm{Bb}$ & $0.69 \mathrm{Be}$ \\
Valencia CNPMF F-11 & $1.00 \mathrm{Ab}$ & $0.83 \mathrm{Aa}$ & $0.84 \mathrm{Aa}$ & $0.83 \mathrm{Ad}$ \\
Valencia Criola & $1.08 \mathrm{Ab}$ & $1.04 \mathrm{Aa}$ & $0.88 \mathrm{Aa}$ & $0.86 \mathrm{Ad}$ \\
Valencia Delta & $0.93 \mathrm{Ac}$ & $0.69 \mathrm{Ab}$ & $0.73 \mathrm{Ab}$ & $0.84 \mathrm{Ad}$ \\
Valencia L. Shaffey & $0.90 \mathrm{Ac}$ & $0.70 \mathrm{Ab}$ & $0.83 \mathrm{Aa}$ & $0.87 \mathrm{Ad}$ \\
Valencia L. White & $1.28 \mathrm{Aa}$ & $0.86 \mathrm{Ba}$ & $0.74 \mathrm{Bb}$ & $0.79 \mathrm{Bd}$ \\
Valencia Late & $1.22 \mathrm{Aa}$ & $0.84 \mathrm{Ba}$ & $0.87 \mathrm{Ba}$ & $0.86 \mathrm{Bd}$ \\
Valencia Midknight & $0.82 \mathrm{Ac}$ & $0.64 \mathrm{Ab}$ & $0.70 \mathrm{Ab}$ & $0.66 \mathrm{Ae}$ \\
Valencia Montemorelos & $1.37 \mathrm{Aa}$ & $0.87 \mathrm{Ba}$ & $0.70 \mathrm{Cb}$ & $0.98 \mathrm{Bc}$ \\
Valencia Registro & $1.43 \mathrm{Aa}$ & $0.87 \mathrm{Ba}$ & $0.90 \mathrm{Ba}$ & $0.86 \mathrm{Bd}$ \\
Valencia Tuxpan & $1.15 \mathrm{Ab}$ & $0.72 \mathrm{Bb}$ & $0.70 \mathrm{Bb}$ & $0.72 \mathrm{Bd}$ \\
Westin & $0.61 \mathrm{Ad}$ & $0.69 \mathrm{Ab}$ & $0.65 \mathrm{Ab}$ & $0.78 \mathrm{Ad}$ \\
\hline
\end{tabular}

Means followed by the same uppercase letter in the row and lowercase in the column do not differ by the Scott-Knott mean cluster test at $5 \%$ probability.

'CNPMF Valencia' cultivar presented higher AT values in the four rootstocks under study. 'Hamlin CNPMF-20', 'Natal CNPMF-02', 'Valencia CNPMF-27', 'Valencia Late', 'Valencia Chapman' and 'Valencia Registro' varieties showed higher fruit acidity levels in $75 \%$ of rootstocks. 'San Diego' and 'Riverside' citrandarins, together, participated in $82.76 \%$ of scion/ rootstock combinations that produced higher-acidity fruits.

AT is considered one of the main variables in identifying the quality and acceptability of citrus fruits by the consumer market. Oranges with desirable citric acid content, AT values must be above $0.5 \%$ and $0.75 \%$ for industrialization and fresh consumption, respectively (RODRIGUES et al., 2019a). Thus, all combinations showed results above $0.75 \%$, placing fruits within the quality standard required by the market.

Scion varieties that produced fruits with the highest SS/AT ratio, with 'Sunki Tropical' mandarin as rootstock were 'Pera CNPMF-01', 'Pera CNPMF B-12', 'Pera CNPMF D-12', 'Pera Bianchi', 'Berna', 'F-Menuda', 'Aquiri', 'Pera de Ibotirama', 'Seleta de Itaborai', 'Westin', 'Crescent sweet' and 'Flor de Brumadinho' (Table 9). In combination with 'San Diego' citrandarin, varieties whose fruits showed relatively high SS/AT ratio were 'Pera CNPMF-01', 'Pera CNPMF D-12', 'Pera CNPMF E-3', 'Pera de Ibotirama', 'Natal Ipeal', 'Melrosa' and 'Flor de Brumadinho'. Still in relation to the SS/AT ratio on 'Riverside' citrandarin, 'Pera CNPMF-02', 'Pera CNPMF E-3', 'Seleta de Itaboraí', 'Natal Ipeal' and 'Flor de Brumadinho' varieties stood out. Finally, in combination with 'Indio' citrandarin, higher SS/AT ratios were identified in 'Pera CNPMF 02', 'Pera CNPMF A-15', 'Pera CNPMF B-12', 'Pera CNPMF D-12' and 'Flor de Brumadinho varieties. Here, there is confirmation of the influence of rootstock on the quality of citrus fruits, in this context in relation to the SS/AT ratio. Regardless of rootstock used, 'Flor de Brumadinho' showed high values for this variable. This trend was also verified in other varieties, such as 'Pera CNPMF D-12'. 
Table 9. SS/AT ratio of fruits from 228 scion/rootstock combinations comprising 57 sweet orange varieties [Citrus sinensis (L.) Osbeck] on 'Sunki Tropical' (TST) mandarin rootstocks [Citrus sunki (Hayata) hort. ex Tanaka] and 'San Diego' (SD), 'Riverside' (RIV) and 'Indio' (IND) citrandarins [C. sunki $\times$ Poncirus trifoliata (L.) Raf.].

\begin{tabular}{|c|c|c|c|c|}
\hline \multirow{2}{*}{ Scion variety } & \multicolumn{4}{|c|}{ Rootstock } \\
\hline & TST & SD & RIV & IND \\
\hline Aquiri & $14.17 \mathrm{Aa}$ & $14.50 \mathrm{Ab}$ & $12.90 \mathrm{Ac}$ & $10.37 \mathrm{Bd}$ \\
\hline Berna & $16.60 \mathrm{Aa}$ & $17.77 \mathrm{Ab}$ & $16.03 \mathrm{Ab}$ & $11.10 \mathrm{Bc}$ \\
\hline Crescent sweet & $13.73 \mathrm{Aa}$ & $16.43 \mathrm{Ab}$ & $16.60 \mathrm{Ab}$ & $15.67 \mathrm{Ab}$ \\
\hline Diva & $11.90 \mathrm{Ab}$ & $12.20 \mathrm{Ac}$ & $13.70 \mathrm{Ac}$ & $14.03 \mathrm{Ab}$ \\
\hline Early Oblong & $10.07 \mathrm{Ac}$ & $12.80 \mathrm{Ac}$ & $11.00 \mathrm{Ac}$ & 10.30 Ad \\
\hline F-Menuda & $14.40 \mathrm{Aa}$ & $11.00 \mathrm{Bc}$ & $14.83 \mathrm{Ab}$ & $8.40 \mathrm{Bd}$ \\
\hline Flor de Brumadinho & $16.37 \mathrm{Ba}$ & $23.77 \mathrm{Aa}$ & $21.50 \mathrm{Aa}$ & 19.97 Aa \\
\hline Hamlin CNPMF 20 & 7.37 Ad & $9.40 \mathrm{Ac}$ & $10.67 \mathrm{Ac}$ & $9.43 \mathrm{Ad}$ \\
\hline Jaffa & $10.33 \mathrm{Bc}$ & 13.67 Ac & $9.50 \mathrm{Bc}$ & $8.63 \mathrm{Bd}$ \\
\hline Mel Rosa & $12.13 \mathrm{Bb}$ & $20.57 \mathrm{Aa}$ & $17.53 \mathrm{Ab}$ & $12.43 \mathrm{Bc}$ \\
\hline Natal CNPMF 01 & $8.50 \mathrm{Ad}$ & $8.57 \mathrm{Ac}$ & $9.20 \mathrm{Ac}$ & $9.10 \mathrm{Ad}$ \\
\hline Natal CNPMF 02 & $7.93 \mathrm{Bd}$ & $9.67 \mathrm{Bc}$ & 12.13 Ac & 7.77 Bd \\
\hline Natal CNPMF 112 & 7.47 Ad & $8.37 \mathrm{Ac}$ & $8.03 \mathrm{Ac}$ & $9.53 \mathrm{Ad}$ \\
\hline Natal Folha Murcha & $8.10 \mathrm{Ad}$ & $11.20 \mathrm{Ac}$ & $10.83 \mathrm{Ac}$ & 9.87 Ad \\
\hline Natal Ipeal & $11.30 \mathrm{Bb}$ & $20.23 \mathrm{Aa}$ & $20.40 \mathrm{Aa}$ & $14.13 \mathrm{Bb}$ \\
\hline Pera Bianchi & $14.67 \mathrm{Aa}$ & $16.47 \mathrm{Ab}$ & $15.10 \mathrm{Ab}$ & $15.00 \mathrm{Ab}$ \\
\hline Pera CNPMF 01 & $13.50 \mathrm{Ba}$ & $20.15 \mathrm{Aa}$ & $17.63 \mathrm{Ab}$ & $13.50 \mathrm{Bb}$ \\
\hline Pera CNPMF 02 & $10.90 \mathrm{Bc}$ & $15.43 \mathrm{Ab}$ & $19.30 \mathrm{Aa}$ & $17.53 \mathrm{Aa}$ \\
\hline Pera CNPMF A-15 & $11.37 \mathrm{Bb}$ & $11.33 \mathrm{Bc}$ & $17.43 \mathrm{Ab}$ & $20.50 \mathrm{Aa}$ \\
\hline Pera CNPMF B-12 & $15.50 \mathrm{Ba}$ & $13.20 \mathrm{Bc}$ & $16.77 \mathrm{Ab}$ & $18.57 \mathrm{Aa}$ \\
\hline Pera CNPMF C-21 & $9.33 \mathrm{Bc}$ & $10.97 \mathrm{Bc}$ & $15.33 \mathrm{Ab}$ & $11.73 \mathrm{Bc}$ \\
\hline Pera CNPMF C-32 & $10.23 \mathrm{Bc}$ & $11.20 \mathrm{Bc}$ & $16.30 \mathrm{Ab}$ & $12.43 \mathrm{Bc}$ \\
\hline Pera CNPMF D-3 & $10.83 \mathrm{Bc}$ & $14.70 \mathrm{Ab}$ & $14.00 \mathrm{Ab}$ & $11.33 \mathrm{Bc}$ \\
\hline Pera CNPMF D-6 & $10.90 \mathrm{Bc}$ & $16.47 \mathrm{Ab}$ & $15.97 \mathrm{Ab}$ & $15.43 \mathrm{Ab}$ \\
\hline Pera CNPMF D-9 & $10.70 \mathrm{Bc}$ & $15.20 \mathrm{Ab}$ & $14.37 \mathrm{Ab}$ & $16.40 \mathrm{Ab}$ \\
\hline Pera CNPMF D-12 & $15.63 \mathrm{Aa}$ & $19.53 \mathrm{Aa}$ & $17.33 \mathrm{Ab}$ & $17.73 \mathrm{Aa}$ \\
\hline Pera CNPMF D-25 & $9.73 \mathrm{Bc}$ & $14.33 \mathrm{Ab}$ & 13.77 Ac & $12.10 \mathrm{Ac}$ \\
\hline Pera CNPMF E-3 & $11.50 \mathrm{Bb}$ & $19.23 \mathrm{Aa}$ & $19.87 \mathrm{Aa}$ & $15.07 \mathrm{Bb}$ \\
\hline Pera CNPMF E-6 & $13.13 \mathrm{Bb}$ & $17.47 \mathrm{Ab}$ & $17.07 \mathrm{Ab}$ & $14.23 \mathrm{Bb}$ \\
\hline Pera GE-03 & $9.40 \mathrm{Bc}$ & $14.90 \mathrm{Ab}$ & $15.47 \mathrm{Ab}$ & $14.13 \mathrm{Ab}$ \\
\hline Pera de Ibotirama & $13.80 \mathrm{Ba}$ & $18.63 \mathrm{Aa}$ & $13.97 \mathrm{Bb}$ & $11.13 \mathrm{Bc}$ \\
\hline Pera Olímpia & $12.67 \mathrm{Bb}$ & $17.60 \mathrm{Ab}$ & $14.53 \mathrm{Bb}$ & $12.80 \mathrm{Bc}$ \\
\hline Pera Vacinada & $12.27 \mathrm{Bb}$ & $16.60 \mathrm{Ab}$ & $14.20 \mathrm{Bb}$ & $12.30 \mathrm{Bc}$ \\
\hline Pineapple & $9.00 \mathrm{Ac}$ & $10.20 \mathrm{Ac}$ & $10.73 \mathrm{Ac}$ & $8.17 \mathrm{Ad}$ \\
\hline Russas P.S. & $11.00 \mathrm{Ac}$ & $11.93 \mathrm{Ac}$ & 11.17 Ac & $10.73 \mathrm{Ac}$ \\
\hline Salustiana & $11.50 \mathrm{Ab}$ & $8.57 \mathrm{Ac}$ & $9.53 \mathrm{Ac}$ & $9.13 \mathrm{Ad}$ \\
\hline
\end{tabular}

continued... 
continuation...

\begin{tabular}{lrrrr}
\hline Seleta de Itaboraí & $14.20 \mathrm{Ba}$ & $14.80 \mathrm{Bb}$ & $21.67 \mathrm{Aa}$ & $14.60 \mathrm{Bb}$ \\
Sincorá & $10.83 \mathrm{Ac}$ & $12.83 \mathrm{Ac}$ & $11.03 \mathrm{Ac}$ & $12.87 \mathrm{Ac}$ \\
Valencia Chapman & $6.40 \mathrm{Ad}$ & $8.57 \mathrm{Ac}$ & $10.20 \mathrm{Ac}$ & $7.87 \mathrm{Ad}$ \\
Valencia CNPMF & $5.90 \mathrm{Bd}$ & $11.27 \mathrm{Ac}$ & $9.97 \mathrm{Ac}$ & $6.17 \mathrm{Bd}$ \\
Valencia CNPMF 01 & $9.93 \mathrm{Bc}$ & $15.07 \mathrm{Ab}$ & $13.17 \mathrm{Ac}$ & $15.83 \mathrm{Ab}$ \\
Valencia CNPMF 02 & $7.70 \mathrm{Bd}$ & $13.07 \mathrm{Ac}$ & $10.10 \mathrm{Bc}$ & $10.23 \mathrm{Bd}$ \\
Valencia CNPMF 03 & 8.17 Ad & $10.53 \mathrm{Ac}$ & $9.90 \mathrm{Ac}$ & $12.07 \mathrm{Ac}$ \\
Valencia CNPMF 21 & $9.17 \mathrm{Ac}$ & $9.93 \mathrm{Ac}$ & $10.37 \mathrm{Ac}$ & $9.23 \mathrm{Ad}$ \\
Valencia CNPMF 27 & $6.90 \mathrm{Ad}$ & $9.27 \mathrm{Ac}$ & $9.83 \mathrm{Ac}$ & $10.40 \mathrm{Ad}$ \\
Valencia CNPMF 36 & 8.53 Bd & $11.53 \mathrm{Ac}$ & $12.23 \mathrm{Ac}$ & $12.73 \mathrm{Ac}$ \\
Valencia CNPMF F-11 & $9.50 \mathrm{Ac}$ & $11.40 \mathrm{Ac}$ & $11.43 \mathrm{Ac}$ & $10.87 \mathrm{Ac}$ \\
Valencia Criola & $7.60 \mathrm{Ad}$ & $9.17 \mathrm{Ac}$ & $10.47 \mathrm{Ac}$ & $10.17 \mathrm{Ad}$ \\
Valencia Delta & $9.07 \mathrm{Ac}$ & $13.13 \mathrm{Ac}$ & $11.53 \mathrm{Ac}$ & $11.67 \mathrm{Ac}$ \\
Valencia L. Shaffey & $9.13 \mathrm{Ac}$ & $12.50 \mathrm{Ac}$ & $11.13 \mathrm{Ac}$ & $10.77 \mathrm{Ac}$ \\
Valencia L. White & $7.23 \mathrm{Bd}$ & $10.37 \mathrm{Ac}$ & $10.67 \mathrm{Ac}$ & $12.43 \mathrm{Ac}$ \\
Valencia Late & $8.03 \mathrm{Ad}$ & $10.67 \mathrm{Ac}$ & $10.00 \mathrm{Ac}$ & $10.93 \mathrm{Ac}$ \\
Valencia Midknight & $10.27 \mathrm{Ac}$ & $12.97 \mathrm{Ac}$ & $12.50 \mathrm{Ac}$ & $12.30 \mathrm{Ac}$ \\
Valencia Montemorelos & $6.67 \mathrm{Bd}$ & $9.37 \mathrm{Ac}$ & $9.57 \mathrm{Ac}$ & $11.10 \mathrm{Ac}$ \\
Valencia Registro & $6.40 \mathrm{Bd}$ & $9.40 \mathrm{Ac}$ & $10.77 \mathrm{Ac}$ & $9.00 \mathrm{Ad}$ \\
Valencia Tuxpan & $7.37 \mathrm{Bd}$ & $11.90 \mathrm{Ac}$ & $11.93 \mathrm{Ac}$ & $11.57 \mathrm{Ac}$ \\
Westin & $15.07 \mathrm{Aa}$ & $14.57 \mathrm{Ab}$ & $14.37 \mathrm{Ab}$ & $12.40 \mathrm{Ac}$ \\
\hline
\end{tabular}

Means followed by the same uppercase letter in the row and lowercase in the column do not differ by the Scott-Knott mean cluster test at $5 \%$ probability.

It is noteworthy that some of scion varieties under study are of low AT, such as 'Berna', 'Aquiri', 'Pera de Ibotirama', 'Seleta de Itaboraí', 'Westin', 'Diva', 'Crescent sweet', 'Melrosa' and 'Flor de Brumadinho'. These materials are accepted for fresh consumption, exclusively, being especially recommended for children, pregnant women and older adults. In addition, fruits from late maturing orange trees, prematurely harvested, like 'Natal' and 'Valencia' clones did not reach their ideal maturation point, yet more than $80 \%$ of the 228 scion/ rootstock combinations showed ratio values equal to or greater than 9.5, which is considered as the minimum required for good quality fruits (CEAGESP, 2011). For the orange juice consumer market, according to Pozzan and Triboni (2005), SS/AT ratio values between 14 and 16 are more acceptable, which are values observed in only $15.78 \%$ of evaluated combinations. In addition, as previously reported, due to the numerous combinations, the maturation curve was not carried out, which will be carried out after selecting the most productive and with better productive efficiency.
Regarding juice yield (REND), it was observed that combinations differed statistically, with the highest yields being observed in 74 combinations, among them 'Pera CNPMF E-6/ 'Riverside' citrandarin, with 58.11\% (Table 10). 
Table 10. Juice yield (\%) of fruits from 228 scion/rootstock combinations comprising 57 sweet orange varieties [Citrus sinensis (L.) Osbeck] on 'Sunki Tropical' (TST) mandarin rootstocks [Citrus sunki (Hayata) hort. ex Tanaka] and 'San Diego' (SD), 'Riverside' (RIV) and 'Indio' (IND) citrandarins [C. sunki $\times$ Poncirus trifoliata (L.) Raf.].

\begin{tabular}{|c|c|c|c|c|}
\hline \multirow{2}{*}{ Scion variety } & \multicolumn{4}{|c|}{ Rootstock } \\
\hline & TST & $\mathrm{SD}$ & RIV & IND \\
\hline Aquiri & 14.17 Aa & $14.50 \mathrm{Ab}$ & $12.90 \mathrm{Ac}$ & $10.37 \mathrm{Bd}$ \\
\hline Berna & $16.60 \mathrm{Aa}$ & $17.77 \mathrm{Ab}$ & $16.03 \mathrm{Ab}$ & $11.10 \mathrm{Bc}$ \\
\hline Crescent sweet & $13.73 \mathrm{Aa}$ & $16.43 \mathrm{Ab}$ & $16.60 \mathrm{Ab}$ & $15.67 \mathrm{Ab}$ \\
\hline Diva & $11.90 \mathrm{Ab}$ & $12.20 \mathrm{Ac}$ & $13.70 \mathrm{Ac}$ & $14.03 \mathrm{Ab}$ \\
\hline Early Oblong & $10.07 \mathrm{Ac}$ & $12.80 \mathrm{Ac}$ & $11.00 \mathrm{Ac}$ & $10.30 \mathrm{Ad}$ \\
\hline F-Menuda & $14.40 \mathrm{Aa}$ & $11.00 \mathrm{Bc}$ & $14.83 \mathrm{Ab}$ & $8.40 \mathrm{Bd}$ \\
\hline Flor de Brumadinho & 16.37 Ba & $23.77 \mathrm{Aa}$ & $21.50 \mathrm{Aa}$ & $19.97 \mathrm{Aa}$ \\
\hline Hamlin CNPMF 20 & 7.37 Ad & $9.40 \mathrm{Ac}$ & $10.67 \mathrm{Ac}$ & $9.43 \mathrm{Ad}$ \\
\hline Jaffa & $10.33 \mathrm{Bc}$ & $13.67 \mathrm{Ac}$ & $9.50 \mathrm{Bc}$ & $8.63 \mathrm{Bd}$ \\
\hline Mel Rosa & $12.13 \mathrm{Bb}$ & $20.57 \mathrm{Aa}$ & $17.53 \mathrm{Ab}$ & $12.43 \mathrm{Bc}$ \\
\hline Natal CNPMF 01 & $8.50 \mathrm{Ad}$ & $8.57 \mathrm{Ac}$ & $9.20 \mathrm{Ac}$ & $9.10 \mathrm{Ad}$ \\
\hline Natal CNPMF 02 & 7.93 Bd & $9.67 \mathrm{Bc}$ & $12.13 \mathrm{Ac}$ & 7.77 Bd \\
\hline Natal CNPMF 112 & 7.47 Ad & $8.37 \mathrm{Ac}$ & $8.03 \mathrm{Ac}$ & $9.53 \mathrm{Ad}$ \\
\hline Natal Folha Murcha & $8.10 \mathrm{Ad}$ & $11.20 \mathrm{Ac}$ & $10.83 \mathrm{Ac}$ & $9.87 \mathrm{Ad}$ \\
\hline Natal Ipeal & $11.30 \mathrm{Bb}$ & $20.23 \mathrm{Aa}$ & $20.40 \mathrm{Aa}$ & $14.13 \mathrm{Bb}$ \\
\hline Pera Bianchi & $14.67 \mathrm{Aa}$ & $16.47 \mathrm{Ab}$ & $15.10 \mathrm{Ab}$ & $15.00 \mathrm{Ab}$ \\
\hline Pera CNPMF 01 & $13.50 \mathrm{Ba}$ & $20.15 \mathrm{Aa}$ & $17.63 \mathrm{Ab}$ & $13.50 \mathrm{Bb}$ \\
\hline Pera CNPMF 02 & $10.90 \mathrm{Bc}$ & $15.43 \mathrm{Ab}$ & $19.30 \mathrm{Aa}$ & $17.53 \mathrm{Aa}$ \\
\hline Pera CNPMF A-15 & $11.37 \mathrm{Bb}$ & $11.33 \mathrm{Bc}$ & $17.43 \mathrm{Ab}$ & $20.50 \mathrm{Aa}$ \\
\hline Pera CNPMF B-12 & $15.50 \mathrm{Ba}$ & $13.20 \mathrm{Bc}$ & $16.77 \mathrm{Ab}$ & $18.57 \mathrm{Aa}$ \\
\hline Pera CNPMF C-21 & $9.33 \mathrm{Bc}$ & $10.97 \mathrm{Bc}$ & $15.33 \mathrm{Ab}$ & $11.73 \mathrm{Bc}$ \\
\hline Pera CNPMF C-32 & $10.23 \mathrm{Bc}$ & $11.20 \mathrm{Bc}$ & $16.30 \mathrm{Ab}$ & $12.43 \mathrm{Bc}$ \\
\hline Pera CNPMF D-3 & $10.83 \mathrm{Bc}$ & $14.70 \mathrm{Ab}$ & $14.00 \mathrm{Ab}$ & $11.33 \mathrm{Bc}$ \\
\hline Pera CNPMF D-6 & $10.90 \mathrm{Bc}$ & $16.47 \mathrm{Ab}$ & $15.97 \mathrm{Ab}$ & $15.43 \mathrm{Ab}$ \\
\hline Pera CNPMF D-9 & $10.70 \mathrm{Bc}$ & $15.20 \mathrm{Ab}$ & $14.37 \mathrm{Ab}$ & $16.40 \mathrm{Ab}$ \\
\hline Pera CNPMF D-12 & $15.63 \mathrm{Aa}$ & $19.53 \mathrm{Aa}$ & $17.33 \mathrm{Ab}$ & $17.73 \mathrm{Aa}$ \\
\hline Pera CNPMF D-25 & $9.73 \mathrm{Bc}$ & $14.33 \mathrm{Ab}$ & $13.77 \mathrm{Ac}$ & $12.10 \mathrm{Ac}$ \\
\hline Pera CNPMF E-3 & $11.50 \mathrm{Bb}$ & $19.23 \mathrm{Aa}$ & $19.87 \mathrm{Aa}$ & $15.07 \mathrm{Bb}$ \\
\hline Pera CNPMF E-6 & $13.13 \mathrm{Bb}$ & $17.47 \mathrm{Ab}$ & $17.07 \mathrm{Ab}$ & $14.23 \mathrm{Bb}$ \\
\hline Pera GE-03 & $9.40 \mathrm{Bc}$ & $14.90 \mathrm{Ab}$ & $15.47 \mathrm{Ab}$ & $14.13 \mathrm{Ab}$ \\
\hline Pera de Ibotirama & $13.80 \mathrm{Ba}$ & $18.63 \mathrm{Aa}$ & $13.97 \mathrm{Bb}$ & $11.13 \mathrm{Bc}$ \\
\hline Pera Olímpia & $12.67 \mathrm{Bb}$ & $17.60 \mathrm{Ab}$ & $14.53 \mathrm{Bb}$ & $12.80 \mathrm{Bc}$ \\
\hline Pera Vacinada & $12.27 \mathrm{Bb}$ & $16.60 \mathrm{Ab}$ & $14.20 \mathrm{Bb}$ & $12.30 \mathrm{Bc}$ \\
\hline Pineapple & $9.00 \mathrm{Ac}$ & $10.20 \mathrm{Ac}$ & $10.73 \mathrm{Ac}$ & $8.17 \mathrm{Ad}$ \\
\hline Russas P.S. & $11.00 \mathrm{Ac}$ & $11.93 \mathrm{Ac}$ & 11.17 Ac & $10.73 \mathrm{Ac}$ \\
\hline Salustiana & $11.50 \mathrm{Ab}$ & $8.57 \mathrm{Ac}$ & $9.53 \mathrm{Ac}$ & $9.13 \mathrm{Ad}$ \\
\hline Seleta de Itaboraí & $14.20 \mathrm{Ba}$ & $14.80 \mathrm{Bb}$ & $21.67 \mathrm{Aa}$ & $14.60 \mathrm{Bb}$ \\
\hline
\end{tabular}


continuation...

\begin{tabular}{lrrrr}
\hline Sincorá & $10.83 \mathrm{Ac}$ & $12.83 \mathrm{Ac}$ & $11.03 \mathrm{Ac}$ & $12.87 \mathrm{Ac}$ \\
Valencia Chapman & $6.40 \mathrm{Ad}$ & $8.57 \mathrm{Ac}$ & $10.20 \mathrm{Ac}$ & $7.87 \mathrm{Ad}$ \\
Valencia CNPMF & $5.90 \mathrm{Bd}$ & $11.27 \mathrm{Ac}$ & $9.97 \mathrm{Ac}$ & $6.17 \mathrm{Bd}$ \\
Valencia CNPMF 01 & $9.93 \mathrm{Bc}$ & $15.07 \mathrm{Ab}$ & $13.17 \mathrm{Ac}$ & $15.83 \mathrm{Ab}$ \\
Valencia CNPMF 02 & $7.70 \mathrm{Bd}$ & $13.07 \mathrm{Ac}$ & $10.10 \mathrm{Bc}$ & $10.23 \mathrm{Bd}$ \\
Valencia CNPMF 03 & $8.17 \mathrm{Ad}$ & $10.53 \mathrm{Ac}$ & $9.90 \mathrm{Ac}$ & $12.07 \mathrm{Ac}$ \\
Valencia CNPMF 21 & $9.17 \mathrm{Ac}$ & $9.93 \mathrm{Ac}$ & $10.37 \mathrm{Ac}$ & $9.23 \mathrm{Ad}$ \\
Valencia CNPMF 27 & $6.90 \mathrm{Ad}$ & $9.27 \mathrm{Ac}$ & $9.83 \mathrm{Ac}$ & $10.40 \mathrm{Ad}$ \\
Valencia CNPMF 36 & $8.53 \mathrm{Bd}$ & $11.53 \mathrm{Ac}$ & $12.23 \mathrm{Ac}$ & $12.73 \mathrm{Ac}$ \\
Valencia CNPMF F-11 & $9.50 \mathrm{Ac}$ & $11.40 \mathrm{Ac}$ & $11.43 \mathrm{Ac}$ & $10.87 \mathrm{Ac}$ \\
Valencia Criola & $7.60 \mathrm{Ad}$ & $9.17 \mathrm{Ac}$ & $10.47 \mathrm{Ac}$ & $10.17 \mathrm{Ad}$ \\
Valencia Delta & $9.07 \mathrm{Ac}$ & $13.13 \mathrm{Ac}$ & $11.53 \mathrm{Ac}$ & $11.67 \mathrm{Ac}$ \\
Valencia L. Shaffey & $9.13 \mathrm{Ac}$ & $12.50 \mathrm{Ac}$ & $11.13 \mathrm{Ac}$ & $10.77 \mathrm{Ac}$ \\
Valencia L. White & $7.23 \mathrm{Bd}$ & $10.37 \mathrm{Ac}$ & $10.67 \mathrm{Ac}$ & $12.43 \mathrm{Ac}$ \\
Valencia Late & $8.03 \mathrm{Ad}$ & $10.67 \mathrm{Ac}$ & $10.00 \mathrm{Ac}$ & $10.93 \mathrm{Ac}$ \\
Valencia Midknight & $10.27 \mathrm{Ac}$ & $12.97 \mathrm{Ac}$ & $12.50 \mathrm{Ac}$ & $12.30 \mathrm{Ac}$ \\
Valencia Montemorelos & $6.67 \mathrm{Bd}$ & $9.37 \mathrm{Ac}$ & $9.57 \mathrm{Ac}$ & $11.10 \mathrm{Ac}$ \\
Valencia Registro & $6.40 \mathrm{Bd}$ & $9.40 \mathrm{Ac}$ & $10.77 \mathrm{Ac}$ & $9.00 \mathrm{Ad}$ \\
Valencia Tuxpan & $7.37 \mathrm{Bd}$ & $11.90 \mathrm{Ac}$ & $11.93 \mathrm{Ac}$ & $11.57 \mathrm{Ac}$ \\
Westin & $15.07 \mathrm{Aa}$ & $14.57 \mathrm{Ab}$ & $14.37 \mathrm{Ab}$ & $12.40 \mathrm{Ac}$ \\
\hline
\end{tabular}

Means followed by the same uppercase letter in the row and lowercase in the column do not differ by the Scott-Knott mean cluster test at $5 \%$ probability.

The combinations under study, except for 'F-Menuda'/ 'Sunki Tropical' mandarin, presented desirable juice yield for fresh consumption, according to the Codex Alimentarius (2005), with values equal to or greater than $35 \%$. However, for Ceagesp (2011), the minimum juice yield for 'Pera', 'Natal', 'Valencia' and 'Hamlin' orange varieties must be, respectively, 45\%, $44 \%$ and $35 \%$. It is noteworthy that for industry use, small variations in this index can lead to large differences in the final juice production.

Although preliminary, the results obtained in this study allowed the identification of promising scion/ rootstock combinations, particularly for citrus crops in the extreme south of the Bahia and Espírito Santo.

Variety from mid-season to late ripening, 'Sincorá' stood out in combination with 'San Diego' and 'Indio' citrandarins in relation to fruit production and juice quality, although it did not present acceptable values in terms of soluble solids content, considering recommendations for fruits intended for fresh consumption.

It should be kept in mind; however, that this is a first fruit crop, a situation that can change in later crops, since fruit quality is lower in the first crops than in more advanced ones.
Among late maturing varieties, such as 'Natal' and 'Valencia' selections harvested in August, the 'Natal Ipeal'/ 'Indio' citrandarin combination stood out for its high efficiency in fruit production and pulp yield, despite the low fruit acidity $(0.69 \%$, citric acid), acceptable for industry, it reached ratio of 14.13, a desirable characteristic for fresh consumption.

Among scion/rootstock combinations that showed high productive efficiency levels, considering those harvested in June, the most prominent were 'Pera CNPMF A-15/ 'San Diego' citrandarin and 'Pera CNPMF D-3/ 'Riverside' citrandarin, which met criteria established by the market regarding fruit quality: ${ }^{\circ}$ Brix of 11.6 and 10.6 , total acidity of $1.04 \%$ and $0.76 \%$, ratio of 11.33 and 14.00 and juice yield of $55.23 \%$ and $50.88 \%$, respectively. However, it was observed that depending on the purpose (industrialization or fresh consumption), growing conditions and harvest time, this indication may vary. 
Considering that the results obtained are preliminary, as well as the place where the study was carried out, the following conclusions are listed below:

1. There are indications that 'Pera CNPMF A-15/ 'San Diego' citrandarin and 'Pera CNPMF D-3/ 'Riverside' citrandarin combinations can be used in the production of fruits for both industry and fresh consumption.

2. Both 'Sincorá' variety in combination with 'San Diego' and 'Indio' citrandarins, and 'Natal Ipeal' variety in combination with 'Indio' citrandarin, present strong indications of the possibility of recommendation to citrus growers, the former being of earlier maturation compared to the latter.

We would like to thank the Espírito Santo Research and Innovation Foundation (FAPES), the Coordination for the Improvement of Higher Education Personnel (CAPES), Bello Fruit - BA and Embrapa Mandioca e Fruticultura.

\section{References}

ALVARES, C.A.; STAPE, J.L.; SENTELHAS, P.C.; GOLÇALVES, J.L.M.; SPAVOREK, G. Köppen's climate classification map for Brazil. Meteorologische Zeitschrift, Stuttgart, v.22, n.6, p.711-728, 2013.

BASTOS, D.C.; FERREIRA, E.A.; PASSOS, O.S.; ATAÍDE, E.; CALGARO, M. Cultivares copa e porta-enxertos para a citricultura brasileira. Informe Agropecuário, Belo Horizonte, v.35, n.281, p.36-45, 2014.

BLUMER, S. Citrandarins e outros híbridos de trifoliata como porta-enxertos nanicantes para a laranjeira 'Valência'(Citrus sinensis L. Osbeck). 2005. $118 \mathrm{f}$. Tese (Doutorado em Agronomia) - Universidade de São Paulo, Piracicaba, 2005.

CARVALHO, L.M. de; CARVALHO, H.W.L. de; SOARES FILHO, W. dos S.; MARTINS, C.R.; PASSOS, O.S. Porta-enxertos promissores, alternativos ao limoeiro 'Cravo', nos Tabuleiros Costeiros de Sergipe. Pesquisa Agropecuária Brasileira, Brasília, DF, v.51, n.2, p.132141, 2016.

CARVALHO, S.A.; GRAF, C.C.D.; VIOLANTE, A.R. Produção de material básico e propagação. In: MATTOS JUNIOR, D.M.; NEGRI, J.D.; PIO, R.M.; POMPEU JUNIOR, J. Citros. Campinas: Instituto Agronômico, Fundag, 2005. p.281-316.
CHAPARRO-ZAMBRANO, H.N.; VELÁSQUEZ, H.A.; ORDUZ-RODRÍGUEZ, J.O. Performance of 'Valencia' sweet orange grafted in different rootstocks, Colombia Tropical Lowland. 2001-2013. Agronomía Colombiana, Bogotá, v.33, p.43-48, 2015.

CEAGESP - Companhia de Entrepostos e Armazéns Gerais de São Paulo. Normas de Classificação de Citros de Mesa. São Paulo: Ceagesp, 2011. 12 p.

COUTO, C.A.; SOUZA, E.R.B; MORGADO, C.M.A.; OGATA, T.; CUNHA JÚNIOR, L.C. Citrus sinensis cultivars: alternatives for diversification of brazilian orchards. Revista Brasileira de Fruticultura, Jaboticabal, v.40, n.5, p.e-097, 2018.

CODEX ALIMENTARIUS. Standard for oranges: Codex Stan 245-2004. 1o Amendment. Rome: FAO e WHO, 2005. 6 p.

DUBEY, A.K. e SHARMA, R.M. Effect of rootstocks on tree growth, yield, quality and leaf mineral composition of lemon (Citrus limon (L.) Burm.). Scientia Horticulturae, New York, v.200, p.131- 136, 2016.

EMBRAPA-Empresa Brasileira de Pesquisa Agropecuária. Manual de análises químicas de solos, plantas e fertilizantes. 2.ed. Brasília: Embrapa Informação Tecnológica, 2009. 627p.

FADEL, A.L.; MOURÃO FILHO, F.D.A.A.; STUCHI, E.S.; WULFF, N.A.; COUTO, H.T.Z. Citrus sudden death-associated virus (CSDaV) and citrus tristeza virus (CTV) in eleven rootstocks for 'Valência'sweet orange. Revista Brasileira de Fruticultura, Jaboticabal, v.40, n.4, p. 1-5, 2018.

FAO. FAOSTAT: food and agriculture. Rome, 2015. Disponível em: http://www.fao.org/statistics/en/\&gt. Acesso em: 23 jul. 2019.

FRANÇA, N. de O.; AMORIM, M. da S.; GIRARDI, E.A.; PASSOS, O.S.; SOARES FILHO, W. dos S. Performance of 'Tuxpan Valencia' sweet orange grafted gnto 14 rootstocks in northern Bahia, Brazil. Revista Brasileira de Fruticultura, Jaboticabal, v.38, n.4, p.1-9, 2016.

IBGE - Instituto Brasileiro de Geografia e Estatística. Levantamento Sistemático da Produção Agrícola, Safra 2019. Rio de Janeiro, 2019. Disponível em: https:// www.ibge.gov.br/estatisticas/economicas/agricultura-epecuaria/9201-levantamento-sistematico-da-producaoagricola.html?=\&t=resultados. Acesso em: 09 mar. 2020. 
IBGE - Instituto Brasileiro de Geografia e Estatística. Levantamento Sistemático da Produção Agrícola, Safra 2019. Rio de Janeiro, 2019. Disponível em: https://sidra. ibge.gov.br/tabela/1618. Acesso em: 01 set. 2020.

LADO, J.; GAMBETTA, G.; ZACARIAS, L. Key determinants of citrus fruit quality: metabolites and main changes during maturation. Scientia Horticulturae, New York, v.233, p.238-248, 2018.

LIMA, C.F. de; MARINHO, C.S.; COSTA, E.S.; ALMEIDA, T.R. de V.; AMARAL, C.O. Qualidade dos frutos e eficiência produtiva da laranjeira 'Lima' enxertada sobre 'Trifloliata', em cultivo irrigado. Revista Brasileira de Ciências Agrárias, Recife, v.9, n.3, p.401-405, 2014.

LIU, X.; LI, J.; HUANG, M.; CHEN, J. Mechanisms for the influence of citrus rootstocks on fruit size. Journal of Agricultural and Food Chemistry, Washington, v.63, p.2618-2627, 2015.

MORETTO, K. M. Estudo sobre uso de novos citrandarins como porta-enxertos para laranja pera. 2019. Dissertação (Mestrado em Produção Vegetal) Universidade Federal de São Carlos, Araras, 2019.

POMPEU JUNIOR, J; BLUMER, S. Híbridos de trifoliata como porta-enxertos para a laranjeira 'Valência'. Pesquisa Agropecuária Brasileira, Brasília, DF, v.44, n.7, p.701705, jul. 2009.

POZZAN, M. E TRIBONI, H. R. Colheita e qualidade do fruto. In: MATTOS JUNIOR, D. de; DE NEGRI, JD; PIO, RM, Citros. Campinas: Instituto Agronômico de Campinas, 2005. p.801-822.

PRUDENTE, R. M.; SILVA, L. M. S. da. Porta-enxerto cítricos. In: MELO, M.B. de; SILVA, L.M.S. de. (ed.). Aspectos técnicos dos citros em Sergipe. Aracaju: Embrapa Tabuleiros Costeiros, 2006.

R Development Core Team. R: A language and environment for statistical computing. R Vienna: Foundation for Statistical Computing, 2011. Disponível em: http://www.R-project.org/.

RODRIGUES, M.J. da S.; ANDRADE NETO, R. de C.; ARAÚJO NETO, S.E. de; SOARES FILHO, W. dos S.; GIRARDI, E.A.; LESSA, L.S.; ALMEIDA, U.O de. Performance of 'Valência' sweet orange grafted onto rootstocks in the state of Acre, Brazil. Pesquisa Agropecuária Brasileira, Brasília, DF, v.54, p.e01349, 2019a.
RODRIGUES, M.J. da S.; ARAÚJO NETO, S.E. de; ANDRADE NETO, R. de C.; SOARES FILHO, W. dos S.; GIRARDI, E.A.; LESSA, L.S.; ALMEIDA, U.O de; ARAUJO J.M. de. Agronomic performance of the 'Pera' orange grafted onto nine rootstocks under the conditions of Rio Branco, Acre, Brazil. Revista Brasileira de Ciências Agrárias, Recife, v.14, n.4, p.e6642, 2019 b.

RODRIGUES, M. J. da S.; DE OLIVEIRA, E. R. M.; GIRARDI, E. A.; LEDO, C. A. D. S.; WALTER, D. S. S. F. Produção de mudas de citros com diferentes combinações copa e porta-enxerto em viveiro protegido. Revista Brasileira de Fruticultura, Jaboticabal, v.38, n.1, p.187-201, 2016.

SANTOS, M.G.; FILHO, W.S.S.; GIRARDI, E.A.; GESTEIRA, A.S.; PASOS, S.O.; FERREIA, C.F. Initial horticultural performance of nine 'Persian' lime selections grafted onto Swingle citrumelo. Scientia Agricola, Piracicaba, v.73, p.109-114, 2016.

SCHAFER, G.; BASTIANEL, M.; DORNELLES, A. L. C. Porta-enxertos utilizados na citricultura, Ciência Rural, Santa Maria, v.31, n.4, p.723-733, 2001.

SOARES, L.A. dos A.; BRITO, M.E.; FERNANDES, P.D.; DE LIMA, G.S.; SOARES FILHO, W dos S.; DE OLIVEIRA, E.S. Crescimento de combinações copa porta-enxerto de citros sob estresse hídrico em casa de vegetação. Revista Brasileira de Engenharia Agrícola e Ambiental, Campina Grande, v.19, n.3, p.211-217, 2015.

SOARES FILHO, W. dos S.; DIAMANTINO, M.S.A.S.; MOITINHO, E.D.B.; SOBRINHO, A.P. da C.; PASSOS, O. S. 'TROPICAL': UMA NOVA SELEÇÃO DE TANGERINA 'SUNKI'. Revista Brasileira de Fruticultura, Jaboticabal, v.24, n.1, p.127-132, 2002.

TARANTINO,A.; LOPS, F.; DISCIGLIO, G.; LOPRIORE, G. Effects of plant biostimulants on fruit set, growth, yield and fruit quality attributes of 'Orange rubis ${ }^{\circledR}$ ' apricot (Prunus armeniaca L.) cultivar in two consecutive years. Scientia Horticulturae, New York, v.239, n.5, p.26-34, 2018.

ZEKRI, M.; OBREZA, T.A; KOO, R. Irrigation, nutrition, and citrus fruit quality. Gainesville: University of Florida, 2003. 3 p. 